\title{
CONVERGENCE OF FINITE DIFFERENCE SCHEMES FOR CONSERVATION LAWS IN SEVERAL SPACE DIMENSIONS: THE CORRECTED ANTIDIFFUSIVE FLUX APPROACH
}

\author{
FREDERIC COQUEL AND PHILIPPE LE FLOCH
}

\begin{abstract}
In this paper, we apply the general method we have presented elsewhere and prove the convergence of a class of explicit and high-order accurate finite difference schemes for scalar nonlinear hyperbolic conservation laws in several space dimensions. We consider schemes constructed-from an $E$-schemeby the corrected antidiffusive flux approach. We derive "sharp" entropy inequalities satisfied by both $E$-schemes and the high-order accurate schemes under consideration. These inequalities yield uniform estimates of the discrete space derivatives of the approximate solutions, which are weaker than the so-called BV (i.e., bounded variation) estimates but sufficient to apply our previous theory.
\end{abstract}

\section{INTRODUCTION}

In this work, we are concerned with the Cauchy problem for nonlinear hyperbolic scalar conservation laws with several space variables:

$$
\partial_{t} u+\partial_{x} \mathbf{f}(u)+\partial_{y} \mathbf{g}(u)=0, \quad u(t, x, y) \in \mathbb{R}, \quad t \in(0, T),(x, y) \in \mathbb{R}^{2},
$$

and

$$
u(0, x, y)=u_{0}(x, y), \quad(x, y) \in \mathbb{R}^{2},
$$

where $\mathbf{f}$ and $\mathbf{g}: \mathbb{R} \rightarrow \mathbb{R}$ are Lipschitz continuous functions and the initial data $u_{0}$ is a given function in $L^{1}\left(\mathbb{R}^{2}\right) \cap L^{\infty}\left(\mathbb{R}^{2}\right)$. As is well known, this problem in general does not admit smooth solutions, so that weak solutions in the sense of distributions must be considered. Moreover, an entropy condition must be added to ensure uniqueness in the class of weak solutions (Lax [27]). Recall also that Volpert [51] has proved the existence and uniqueness of such an entropy weak solution in the class of bounded functions of bounded variation, and Kružkov [22] has extended the result of Volpert to the more general class of bounded functions.

This paper treats the approximation of the entropy weak solution to problem (1.1), (1.2) by high-order accurate and explicit finite difference schemes. A

Received January 26, 1990.

1980 Mathematics Subject Classification (1985 Revision). Primary 35L65, 65N10. 
previous work (Coquel and Le Floch [7]) has presented a general theory to prove convergence of finite difference schemes for equations in several space dimensions. In the present work, we apply the method of [7] to get the convergence of the schemes constructed by the so-called corrected antidiffusive flux approach.

Let us first recall that the classical approach used to show convergence of finite difference schemes for scalar conservation laws is based on the Lax-Wendroff Theorem $[28,29]$. This requires both a uniform $L^{\infty}$-bound and strong $L^{1}$ convergence of the family of approximate solutions. On the other hand, as is well known, in the case that a uniform estimate of the total variation of the approximate solutions, i.e., a $B V$-estimate, is available, the compactness theorem of Helly gives strong $L^{1}$-convergence of the approximate solutions. Actually, this argument yields convergence of some high-order accurate difference schemes in the case of equations in only one space dimension, but convergence of only first-order accurate schemes for equations with several space variables. Concerning the techniques of derivation of BV-estimates, we refer among others to the works of Crandall and Majda [8], Harten [21], Leroux [30, 31], Osher [35], Osher and Chakravarthy [36], Sanders [37], Shu [39], Sweby [42], and Tadmor [45].

In contrast with this approach, the theory of Coquel and Le Floch [7] allows one to prove convergence of finite difference schemes without using a uniform $\mathrm{BV}$-estimate. The method of proof is based on a uniqueness result in the class of entropy measure-valued solutions, due to Di Perna [15]. In [7], a certain "weak estimate" of the space derivatives of the approximate solutions is introduced, but this estimate indeed turns out to be weaker than the usual BV-estimate. General convergence theorems which generalize the Lax-Wendroff Theorem are proved in [7] in the setting of measure-valued solutions. These theorems of convergence, combined with the uniqueness theorem of Di Perna, yield strong $L^{1}$-convergence of the sequence of approximate solutions.

This paper is devoted to the application of this theory to a class of finite difference schemes. To be specific, we focus on the high-order accurate schemes which are constructed from an $E$-scheme (Osher [35]) by the corrected antidiffusive flux approach and, for the sake of simplicity, we restrict ourselves to the schemes defined on regular Cartesian meshes. Actually, the method of proof presented here is very general and could be applied to the case of irregular meshes or to implicit schemes, as well as finite volume schemes. Moreover, we present our results for an equation with two space variables, but the extension to an arbitrary number of space variables is immediate.

The results of convergence known for the schemes with corrected antidiffusive flux concern only equations in one space dimension: for instance, Cahouet and Coquel [3], Leroux and Quesseveur [32], and Vila [50]. The main result of this paper is a generalization of these results of convergence to the case of equations with several space variables. Moreover, even in the case of equations with one space variable, our method turns out to improve existing results.

In order to apply the method of proof of [7], the main difficulty is to derive a "weak estimate" of the space derivatives of the approximate solutions. Recall 
that this estimate replaces the usual BV-estimate and is weaker than this latter (see [7]). For this purpose, we obtain in this paper "sharp" entropy inequalities (in a sense specified in $\S 3$ below) satisfied by the schemes under consideration, i.e., both $E$-schemes and the high-order schemes with corrected antidiffusive flux. We emphasize that the above inequality is derived from a sharp evaluation of the entropy dissipation of the schemes, and the required uniform estimate of the space derivatives is precisely provided by the term of entropy dissipation $([7, \S 2]$ and $\S 4$ below).

An outline of the paper is as follows. Section 2 recalls the general method of proof. The derivation of the sharp entropy inequalities satisfied by certain firstorder schemes is done in $\S 3$. Finally, $\S 4$ generalizes the inequalities obtained in $\S 3$ to the high-order accurate schemes with corrected antidiffusive flux and gives the proof of convergence. Throughout the paper, $C$ denotes a positive constant independent of the increments of the discretization and is not necessarily the same at each occurrence.

\section{SURVEY OF THE GENERAL THEORY}

This section gives the main results of [7] which will be needed in this paper. We refer to [7] for the details and the proofs. The main tool used in our approach is the concept of measure-valued solutions. Instead of functions in $L^{\infty}\left((0, T) \times \mathbb{R}^{2}\right)$, we consider Young measures on $(0, T) \times \mathbb{R}^{2}$, that is, weakstar bounded applications $\nu:(0, T) \times \mathbb{R}^{2} \rightarrow \operatorname{Prob}(\mathbb{R})$, where $\operatorname{Prob}(\mathbb{R})$ is the space of all measures of probability on $\mathbb{R}$, i.e., nonnegative measures with unit total mass. As in [15] and [7], all the Young measures will be tacitly assumed to be supported in a compact set $K$ of $\mathbb{R}$. We recall from Di Perna [15] that a Young measure $\nu:(0, T) \times \mathbb{R}^{2} \rightarrow \operatorname{Prob}(\mathbb{R})$ is a measure-valued solution to the conservation law (1.1) if it satisfies

$$
\partial_{t}\langle\nu, \mathrm{id}\rangle+\partial_{x}\langle\nu, \mathbf{f}\rangle+\partial_{y}\langle\nu, \mathbf{g}\rangle=0
$$

in the sense of distributions. This definition indeed generalizes the usual notion of weak $L^{\infty}$-solutions in the sense of distributions (see [15]). Moreover, for the sake of uniqueness, it is necessary to add an entropy condition (Lax [27]). Recall that a Lipschitz continuous function $(\mathbf{U}, \mathbf{F}, \mathbf{G}): \mathbb{R} \rightarrow \mathbb{R}^{3}$ is said to be an entropy for equation (1.1) if $\mathbf{U}$ is a convex function and $\mathbf{F}$ and $\mathbf{G}$ satisfy the following compatibility relations:

$$
\mathbf{U}^{\prime}(v) \mathbf{f}^{\prime}(v)=\mathbf{F}^{\prime}(v), \quad \mathbf{U}^{\prime}(v) \mathbf{g}^{\prime}(v)=\mathbf{G}^{\prime}(v), \quad \text { a.e. } v \in \mathbb{R}
$$

Such a function $(\mathbf{U}, \mathbf{F}, \mathbf{G})$ corresponds to an additional conservation law satisfied by each smooth solution of (1.1). A Young measure $\nu$ is consistent with the entropy inequality associated with $(\mathbf{U}, \mathbf{F}, \mathbf{G})$ if it satisfies

$$
\partial_{t}\langle\nu, \mathbf{U}\rangle+\partial_{x}\langle\nu, \mathbf{F}\rangle+\partial_{y}\langle\nu, \mathbf{G}\rangle \leq 0
$$

in the sense of distributions. A measure-valued solution $\nu$ is an entropy measure-valued solution if it satisfies $(2.3)$ for all entropies $(\mathbf{U}, \mathbf{F}, \mathbf{G})$. 
In the class of entropy measure-valued solutions, Di Perna has proved the following generalization of the Kružkov uniqueness theorem [22].

Theorem 2.1 (Di Perna [15]). Let the initial data $u_{0}$ be in $L^{1}\left(\mathbb{R}^{2}\right) \cap L^{\infty}\left(\mathbb{R}^{2}\right)$. Assume that $\nu:(0, T) \times \mathbb{R}^{2} \rightarrow \operatorname{Prob}(\mathbb{R})$ is a Young measure which satisfies the following properties:

(1) $\nu$ is a measure-valued solution to equation (1.1),

(2) $\nu$ is consistent with all entropy inequalities (2.3),

(3) $\nu$ assumes its initial data $u_{0}$ in the following sense:

(2.4) the function $(t, x, y) \rightarrow\left\langle\nu_{t, x, y},\right|$ id |\rangle belongs to $L^{\infty}\left((0, T), L^{1}\left(\mathbb{R}^{2}\right)\right)$, and

$$
\lim _{\substack{t \rightarrow 0 \\ t>0}} \frac{1}{t} \int_{0}^{t} \int_{\mathbb{R}^{2}}\left\langle\nu_{s, x, y},\left|\mathrm{id}-u_{0}(x, y)\right|\right\rangle d x d y d s=0 .
$$

Then, if $u$ in $L^{\infty}\left((0, T) \times \mathbb{R}^{2}\right)$ denotes the unique entropy weak $L^{\infty}$-solution to problem (1.1), (1.2), we have

$$
\nu_{t, x, y}=\delta_{u(t, x, y)} \text {, a.e. }(t, x, y) \in \mathbb{R}^{2} .
$$

We now consider approximate solutions to (1.1), (1.2) constructed by general explicit finite difference schemes, and we recall several general convergence theorems proved in [7] which are useful to apply Theorem 2.1 in the context of finite difference schemes. Let us first introduce some notation. Let $h, h_{x}$, and $h_{y}$ be the time, $x$-space, and $y$-space increments of the discretization, respectively. The mesh ratios

$$
\lambda_{x}=\frac{h}{h_{x}}, \quad \lambda_{y}=\frac{h}{h_{y}}
$$

will be kept constant and should satisfy a Courant-Friedrichs-Levy (CFL) stability condition. We define a regular grid by setting

$$
t_{n}=n h \quad(n \in \mathbb{N}), \quad x_{i}=i h_{x}, \quad y_{j}=j h_{y} \quad\left(i, j \in \mathbb{Z} \text { or } i, j \in \mathbb{Z}+\frac{1}{2}\right) .
$$

We consider approximate solutions $u^{h}: \mathbb{R}_{+} \times \mathbb{R}^{2} \rightarrow \mathbb{R}$ to problem (1.1), (1.2) which are piecewise constant, i.e., for $t \in\left[t_{n}, t_{n+1}\right)$

$$
u^{h}(t, x, y)=u_{i j}^{n}, \quad x \in\left[x_{i-1 / 2}, x_{i+1 / 2}\right), y \in\left[y_{j-1 / 2}, y_{j+1 / 2}\right) .
$$

For $t=t_{0}=0,\left(u_{i, j}^{0}\right)_{i, j \in \mathbb{Z}}$ is defined from the initial data $u_{0}$ by projection,

$$
u_{i, j}^{0}=\frac{1}{h_{x} h_{y}} \int_{x_{i-1 / 2}}^{x_{i+1 / 2}} \int_{y_{j-1 / 2}}^{y_{j+1 / 2}} u_{0}(x, y) d x d y, \quad i, j \in \mathbb{Z} .
$$

Then for each integer $n$, the sequence $\left(u_{i, j}^{n+1}\right)_{i, j \in \mathbb{Z}}$ is given by the following $(2 k+1)$-point explicit difference scheme: for any $i, j \in \mathbb{Z}$

$$
u_{i, j}^{n+1}=u_{i, j}^{n}-\lambda_{x}\left(f_{i+1 / 2, j}^{n}-f_{i-1 / 2, j}^{n}\right)-\lambda_{y}\left(g_{i, j+1 / 2}^{n}-g_{i, j-1 / 2}^{n}\right),
$$


where $f_{i+1 / 2, j}^{n}$ and $g_{i, j+1 / 2}^{n}$ are defined from two given numerical flux functions $f$ and $g: \mathbb{R}^{2 k} \rightarrow \mathbb{R}$ by

$$
\begin{array}{ll}
f_{i+1 / 2, j}^{n}=f\left(u_{i-k+1, j}^{n}, u_{i-k+2, j}^{n}, \ldots, u_{i+k, j}^{n}\right), & i, j \in \mathbb{Z}, \\
g_{i, j+1 / 2}^{n}=g\left(u_{i, j-k+1}^{n}, u_{i, j-k+2}^{n}, \ldots, u_{i, j+k}^{n}\right), & i, j \in \mathbb{Z} .
\end{array}
$$

As usual, the functions $f$ and $g$ are assumed to be consistent with the exact flux functions $\mathbf{f}$ and $\mathbf{g}$, i.e., they are Lipschitz continuous and satisfy

$$
f(v, v, \ldots, v)=\mathbf{f}(v), \quad g(v, v, \ldots, v)=\mathbf{g}(v), \quad v \in \mathbb{R} .
$$

Assuming that the family of approximate solutions $\left\{u^{h}\right\}$ defined by (2.9)-(2.12) is uniformly bounded in $L^{\infty}$-norm,

$$
\left\|u^{h}\right\|_{L^{\infty}\left((0, T) \times \mathbb{R}^{2}\right)} \leq C,
$$

one can construct a Young measure $\nu$ to represent all the $L^{\infty}$-weak-star composite limits of $u^{h}$ when $h$ (or a subsequence) tends to zero. In other words, we have

$$
a\left(u^{h}\right) \rightarrow\langle\nu, a\rangle, \quad \text { weak-star in } L^{\infty}\left((0, T) \times \mathbb{R}^{2}\right),
$$

for each continuous function $a: \mathbb{R} \rightarrow \mathbb{R}$ (Tartar [47, 48]; see also Ball [1] and Dacorogna [9]). The following result proved in Coquel and Le Floch [7] is an extension of the Lax-Wendroff Theorem [28, 29].

Theorem 2.2. Assume that the numerical flux functions $f$ and $g$ are consistent with equation (1.1) and the family of approximate solutions $\left\{u^{h}\right\}$ defined by the difference scheme (2.9)-(2.12) satisfies the following properties:

(1) the uniform $L^{\infty}$-estimate (2.14),

(2) there exists $\beta$ in $[0,1)$ such that

$$
h^{\beta} \sum_{\substack{n \in \mathbb{N} \\ n h \leq T}} \sum_{i, j \in \mathbb{Z}}\left\{\frac{\left|u_{i+1, j}^{n}-u_{i, j}^{n}\right|}{h_{x}}+\frac{\left|u_{i, j+1}^{n}-u_{i, j}^{n}\right|}{h_{y}}\right\} h_{x} h_{y} h \leq C,
$$

where the constant $C$ is independent of $h$ (with $\lambda_{x}=h / h_{x}$ and $\lambda_{y}=h / h_{y}$ kept constant). Then the Young measure $\nu$ associated with $\left\{u^{h}\right\}$ is a measure-valued solution to (1.1).

Assumption (2.15) is a uniform estimate of the discrete space derivatives of $\left\{u^{h}\right\}$ that will be called the weak estimate of the space derivatives. For $\beta \in(0,1)$, estimate $(2.15)$ is indeed weaker than a BV-estimate because of the weight $h^{\beta}$. Several comments concerning (2.15) can be found in [7].

Let $(\mathbf{U}, \mathbf{F}, \mathbf{G})$ be an entropy for equation (1.1); a function $(\mathbf{U}, F, G)$ is a numerical entropy consistent with the (exact) entropy $(\mathbf{U}, \mathbf{F}, \mathbf{G})$ if the functions $F$ and $G$ are Lipschitz-continuous functions defined from $\mathbb{R}^{2 k}$ into $\mathbb{R}$ and satisfy

$$
F(v, v, \ldots, v)=\mathbf{F}(v), \quad G(v, v, \ldots, v)=\mathbf{G}(v), \quad v \in \mathbb{R} .
$$


For simplicity, we set

$$
\left\{\begin{array}{l}
U_{i j}^{n}=\mathbf{U}\left(u_{i j}^{n}\right), \\
F_{i+1 / 2, j}^{n}=F\left(u_{i-k+1, j}^{n}, \ldots, u_{i+k, j}^{n}\right), \\
G_{i, j+1 / 2}^{n}=G\left(u_{i, j-k+1}^{n}, \ldots, u_{i, j+k}^{n}\right), \quad n \in \mathbb{N}, i, j \in \mathbb{Z} .
\end{array}\right.
$$

The following theorem is proved in [7].

Theorem 2.3. Assume that the hypotheses (2.13)-(2.15) of Theorem 2.2 are satisfied. Let $(\mathbf{U}, F, G)$ be a numerical entropy consistent with an exact entropy $(\mathbf{U}, \mathbf{F}, \mathbf{G})$ for equation (1.1). Suppose that the family of approximate solutions $\left\{u^{h}\right\}$ satisfies the following inequality $(n \in \mathbb{N}, i, j \in \mathbb{Z})$ :

(2.16) $\frac{1}{h}\left(U_{i, j}^{n+1}-U_{i, j}^{n}\right)+\frac{1}{h_{x}}\left(F_{i+1 / 2, j}^{n}-F_{i-1 / 2, j}^{n}\right)+\frac{1}{h_{y}}\left(G_{i, j+1 / 2}^{n}-G_{i, j-1 / 2}^{n}\right) \leq R_{i, j}^{n}$,

where the term $R_{i, j}^{n}$ tends to zero in the following weak sense:

$$
\sum_{\substack{n \in \mathbb{N} \\ n h \leq T}} \sum_{i, j \in \mathbb{Z}} \varphi\left(t_{n}, x_{i}, y_{j}\right) R_{i, j}^{n} h_{x} h_{y} h \rightarrow 0, \quad \text { when } h \rightarrow 0,
$$

for every function $\varphi:(0, T) \times \mathbb{R}^{2} \rightarrow \mathbb{R}_{+}$of class $C^{1}$ with compact support $\left(\lambda_{x}=h / h_{x}\right.$ and $\lambda_{y}=h / h_{y}$ are kept constant $)$. Then the measure-valued solution $\nu$ associated with $\left\{u^{h}\right\}$ is consistent with the entropy $(\mathbf{U}, \mathbf{F}, \mathbf{G})$. Moreover, if $\left\{u^{h}\right\}$ satisfies (2.16), (2.17) for all entropy ( $\left.\mathbf{U}, \mathbf{F}, \mathbf{G}\right)$, then $\nu$ is an entropy measure-valued solution to (1.1).

Concerning the initial condition (1.2), one has [7]:

Theorem 2.4. Let the initial data $u_{0}$ be in $L^{1}\left(\mathbb{R}^{2}\right) \cap L^{\infty}\left(\mathbb{R}^{2}\right)$. Assume that the sequence of approximate solutions $\left\{u^{h}\right\}$ satisfies (2.14), (2.15) and the following uniform $L^{1}$-estimate:

$$
\left\|u^{h}(t, \cdot, \cdot)\right\|_{L^{1}\left(\mathbb{R}^{2}\right)} \leq C, \text { a.e. } t>0 .
$$

Suppose moreover that, for one given strictly convex entropy $\mathbf{U}$ satisfying $\mathbf{U}(0)=$ 0 , the discrete entropy inequality (2.16) holds with

$$
\lim _{\substack{h \rightarrow 0 \\ h>0}} \sum_{\substack{n \in \mathbb{N} \\ n h \leq T}} \sum_{i, j \in \mathbb{Z}}\left|R_{i, j}^{n}\right| h_{x} h_{y} h \rightarrow 0 \text { as } t \text { goes to } 0 .
$$

Then the Young measure $\nu$ associated with the family $\left\{u^{h}\right\}$ satisfies the initial condition (1.2) in the sense (2.4), (2.5).

Theorems 2.2, 2.3, and 2.4 show that the Young measure $\nu$ associated with the family of approximate solutions $\left\{u^{h}\right\}$ is an entropy measure-valued solution to (1.1) which satisfies the initial condition (1.2) in the strong sense. Thus, by Theorem 2.1, $\nu$ is a Dirac mass, i.e., (2.6) holds. In that case, we can conclude that $u^{h}$ converges strongly in $L^{1}$ to the unique entropy weak $L^{\infty}$-solution to 
problem (1.1), (1.2). This method of proof will be applied in $\S 4$ of this paper to show convergence of the schemes constructed by the corrected antidiffusive flux approach. Recall also that the above method generalizes to finite difference schemes a result of Szepessy [43, 44] which concerns the streamline diffusion finite element method.

\section{A SHARP ENTROPY INEQUALITY SATISFIED BY $E$-SCHEMES}

3.1. Introduction. In this section, we derive a sharp entropy inequality for (firstorder accurate) $E$-schemes applied to an equation with one space variable. This inequality is fundamental for the proofs of convergence of the next section and, in particular, will be generalized there to equations with several space variables and to high-order accurate schemes constructed from $E$-schemes.

Entropy inequalities for, say, first-order accurate schemes like $E$-schemes have already been obtained in the literature (see, for instance, Crandall and Majda [8], Osher [35], Tadmor [45],... ). But in the present work, we are interested in a stronger version of these inequalities, that we call "sharp" entropy inequalities. They are of interest since they yield an estimate of the discrete space derivatives of the approximate solutions, i.e., precisely an estimate of the form (2.15). About such sharp entropy inequalities, only partial results have been obtained in the literature, mainly by authors concerned with applications of the method of compensated compactness. Some ideas of the proofs below come from the work of Di Perna [13]; we also mention Lax [26], Ding, Chen, and Luo [12], and Wang, Li, and Huang [52]. On the other hand, we emphasize that our results below give the precise CFL-like stability conditions and probably better estimates of that sort. In particular, we point out a substantial difference between the Godunov scheme and the modified Lax-Friedrichs scheme [45]: the rate of entropy dissipation (see below) turns out to be cubic for the former and quadratic for the latter.

Here is an outline of this section. We first consider in $\S 3.2$ the Godunov scheme and then in $\S 3.3$ the modified Lax-Friedrichs scheme. The extension to a general $E$-scheme is obtained in $\S 3.4$ by writing it as a convex combination of the Godunov scheme and the modified Lax-Friedrichs scheme. Finally, $\S 3.5$ is concerned with the class of schemes whose numerical viscosity coefficient is bounded below by a positive constant.

In this section, since we consider an equation with only one space variable, we use the notation introduced in $\S 2$ except that the letters $y, j$, etc. are omitted. The given flux function $\mathbf{f}$ is assumed to be of $C^{2}$ class and uniformly convex (several remarks concerning the case of a not necessarily convex function are also given in this section). Our estimates will depend explicitly on the convexity modulus of the function $f$ given by

$$
\delta=\inf _{u} \mathbf{f}^{\prime \prime}(u),
$$

the infimum being taken over all $u$ under consideration. The sonic point $u_{*}$ is 
defined by

$$
\mathbf{f}^{\prime}\left(u_{*}\right)=0
$$

For the sake of simplicity in the presentation (it is not a restriction for our purpose, and the extension to a general entropy is immediate), we shall use throughout this section the entropy $(\mathbf{U}, \mathbf{F})$ defined by

$$
\mathbf{U}(u)=\frac{u^{2}}{2}, \quad \mathbf{F}(u)=\int_{0}^{u} v \mathbf{f}^{\prime}(v) d v, \quad \forall u \in \mathbb{R} .
$$

3.2. A cubic estimate of the entropy dissipation in the Godunov scheme. For any $u_{L}$ and $u_{R}$ in $\mathbb{R}$, let $\frac{x}{t} \rightarrow w\left(\frac{x}{t} ; u_{L}, u_{R}\right)$ denote the unique entropy weak solution to the Riemann problem

$$
\partial_{t} u+\partial_{x} \mathbf{f}(u)=0, \quad t>0, x \in \mathbb{R},
$$

and

$$
u(0, x)= \begin{cases}u_{L} & \text { for } x<0 \\ u_{R} & \text { for } x>0\end{cases}
$$

Since the function $\mathbf{f}$ is strictly convex, $w\left(\cdot ; u_{L}, u_{R}\right)$ is composed of either a shock wave (if $u_{L}>u_{R}$ ) or a rarefaction wave (if $u_{L} \leq u_{R}$ ) (see Lax [27]). Then, the Godunov scheme is defined by its numerical flux function $f_{G}$ given by

$$
f_{G}\left(u_{L}, u_{R}\right)=\mathbf{f}\left(w\left(0 ; u_{L}, u_{R}\right)\right), \quad u_{L}, u_{R} \in \mathbb{R} .
$$

We assume that the CFL stability restriction on $\lambda_{x}$,

$$
\lambda_{x} \sup _{u}\left|\mathbf{f}^{\prime}(u)\right| \leq \frac{1}{2}
$$

is satisfied (the supremum is taken over all $u$ under consideration) and will use an averaged form of the Godunov scheme introduced by Tadmor [45]. Following the notation of $\S 2$, the sequence $\left(u_{i}^{n}\right)_{n \in \mathbb{N}, i \in \mathbb{Z}}$ constructed by the Godunov scheme can be redefined by

$$
u_{i}^{n+1}=\frac{1}{2}\left(u_{i-1 / 2}^{n+1, R}+u_{i+1 / 2}^{n+1, L}\right), \quad n \in \mathbb{N}, i \in \mathbb{Z},
$$

where we have set

$$
u_{i-1 / 2}^{n+1, R}=\frac{2}{h_{x}} \int_{0}^{h_{x} / 2} w\left(\frac{x}{h} ; u_{i-1}^{n}, u_{i}^{n}\right) d x, \quad n \in \mathbb{N}, i \in \mathbb{Z},
$$

and

$$
u_{i+1 / 2}^{n+1, R}=\frac{2}{h_{x}} \int_{-h_{x} / 2}^{0} w\left(\frac{x}{h} ; u_{i}^{n}, u_{i+1}^{n}\right) d x, \quad n \in \mathbb{N}, i \in \mathbb{Z} .
$$

This decomposition of the Godunov scheme is helpful to derive the entropy inequalities below. Recall also that the function

$$
F_{G}\left(u_{L}, u_{R}\right)=\mathbf{F}\left(w\left(0+; u_{L}, u_{R}\right)\right), \quad u_{L}, u_{R} \in \mathbb{R},
$$


is a numerical entropy-flux for the Godunov scheme associated with the given (quadratic) entropy (3.3). As is usual, we set

$$
F_{G, i+1 / 2}^{n}=F_{G}\left(u_{i}^{n}, u_{i+1}^{n}\right), \quad n \in \mathbb{N}, i \in \mathbb{Z} .
$$

Then, following an idea introduced by Di Perna in [13], we are able to give a closed formula for the dissipation of entropy in each subcell of the form $[n h,(n+1) h) \times\left[(i+1 / 2) h_{x},(i+1) h_{x}\right)$ or $[n h,(n+1) h) \times\left[i h_{x},(i+1 / 2) h_{x}\right)$ $(n \in \mathbb{N}, i \in \mathbb{Z})$.

Lemma 3.1 (Godunov scheme). Consider the Godunov scheme (3.6) under the CFL condition (3.5). Then, for each $n$ in $\mathbb{N}$ and each $i$ in $\mathbb{Z}$, we have

$$
\begin{aligned}
& \mathbf{U}\left(u_{i+1 / 2}^{n+1, R}\right)-\mathbf{U}\left(u_{i+1}^{n}\right)+2 \lambda_{x}\left(\mathbf{F}\left(u_{i+1}^{n}\right)-F_{G, i+1 / 2}^{n}\right) \\
& \quad=2 \lambda_{x} J_{i+1 / 2}^{n, R}-\frac{1}{h_{x}} \int_{0}^{h_{x} / 2}\left|w\left(\frac{x}{h} ; u_{i}^{n}, u_{i+1}^{n}\right)-u_{i+1 / 2}^{n+1, R}\right|^{2} d x
\end{aligned}
$$

and

$$
\begin{aligned}
& \mathbf{U}\left(u_{i+1 / 2}^{n+1, L}\right)-\mathbf{U}\left(u_{i}^{n}\right)-2 \lambda_{x}\left(\mathbf{F}\left(u_{i}^{n}\right)-F_{G, i+1 / 2}^{n}\right) \\
& \quad=2 \lambda_{x} J_{i+1 / 2}^{n, L}-\frac{1}{h_{x}} \int_{-h_{x} / 2}^{0}\left|w\left(\frac{x}{h} ; u_{i}^{n}, u_{i+1}^{n}\right)-u_{i+1 / 2}^{n+1, L}\right|^{2} d x,
\end{aligned}
$$

where the terms $J_{i+1 / 2}^{n, R}$ and $J_{i+1 / 2}^{n, L}$ equal zero except when the Riemann solution $w\left(\cdot ; u_{i}^{n}, u_{i+1}^{n}\right)$ contains a shock wave with speed $\sigma_{i+1 / 2}^{n}$ and, in this latter case, they are given by

$$
J_{i+1 / 2}^{n, R}=\left\{\begin{array}{l}
\mathbf{F}\left(u_{i+1}^{n}\right)-\mathbf{F}\left(u_{i}^{n}\right)-\sigma_{i+1 / 2}^{n}\left(\mathbf{U}\left(u_{i+1}^{n}\right)-\mathbf{U}\left(u_{i}^{n}\right)\right), \quad \text { if } \sigma_{i+1 / 2}^{n}>0 \\
0, \quad \text { otherwise }
\end{array}\right.
$$

and

$$
J_{i+1 / 2}^{n, L}=\left\{\begin{array}{l}
\mathbf{F}\left(u_{i+1}^{n}\right)-\mathbf{F}\left(u_{i}^{n}\right)-\sigma_{i+1 / 2}^{n}\left(\mathbf{U}\left(u_{i+1}^{n}\right)-\mathbf{U}\left(u_{i}^{n}\right)\right), \quad \text { if } \sigma_{i+1 / 2}^{n} \leq 0 \\
0, \quad \text { otherwise. }
\end{array}\right.
$$

Remark 3.1. In the case that the Riemann solution $w\left(\cdot ; u_{i}^{n}, u_{i+1}^{n}\right)$ consists of a shock wave with zero speed $\left(\sigma_{i+1 / 2}^{n}=0\right)$, then by definition the term $J_{i+1 / 2}^{n, L}$ equals the amount of entropy dissipation associated with this stationary shock wave, i.e.,

$$
J_{i+1 / 2}^{n, L}=\mathbf{F}\left(u_{i+1}^{n}\right)-\mathbf{F}\left(u_{i}^{n}\right) \quad(\neq 0 \text { in general }),
$$

while we have $J_{i+1 / 2}^{n, R}=0$. This is due to the definition (3.7) of the numerical entropy-flux function.

Proof of Lemma 3.1. We only prove formula (3.8a); the proof of (3.8b) is similar. In any zone of smoothness of the function $w\left(\cdot ; u_{i}^{n}, u_{i+1}^{n}\right)$, we have the conservation law of entropy

$$
\partial_{t} \mathbf{U}\left(w\left(\cdot ; u_{i}^{n}, u_{i+1}^{n}\right)\right)+\partial_{x} \mathbf{F}\left(w\left(\cdot ; u_{i}^{n}, u_{i+1}^{n}\right)\right)=0
$$


Thus, by integration over each domain of regularity of the subcell $(0, h) \times$ $\left(0, h_{x} / 2\right)$ and under the CFL condition (3.5), we obtain

$$
\begin{aligned}
& \int_{0}^{h_{x} / 2} \mathbf{U}\left(w\left(\frac{x}{h} ; u_{i}^{n}, u_{i+1}^{n}\right)\right) d x-\frac{h_{x}}{2} \mathbf{U}\left(u_{i+1}^{n}\right) \\
& \quad+h\left\{\mathbf{F}\left(u_{i+1}^{n}\right)-\mathbf{F}\left(w\left(0+; u_{i}^{n}, u_{i+1}^{n}\right)\right)\right\}=h J_{i+1 / 2}^{n, R},
\end{aligned}
$$

with the jump term defined by $(3.9 \mathrm{a})$. We then observe that

$$
\begin{aligned}
& \mathbf{U}\left(w\left(\cdot ; u_{i}^{n}, u_{i+1}^{n}\right)\right)-\mathbf{U}\left(u_{i+1 / 2}^{n+1, R}\right)-u_{i+1 / 2}^{n+1, R}\left(w\left(\cdot ; u_{i}^{n}, u_{i+1}^{n}\right)-u_{i+1 / 2}^{n+1, R}\right) \\
& \quad=\frac{1}{2}\left|w\left(\cdot ; u_{i}^{n}, u_{i+1}^{n}\right)-u_{i+1 / 2}^{n+1, R}\right|^{2}
\end{aligned}
$$

since $\mathbf{U}(u)=u^{2} / 2$. Hence, by (3.6b) we have

$$
\begin{aligned}
& \frac{2}{h_{x}} \int_{0}^{h_{x} / 2} \mathbf{U}\left(w\left(\cdot ; u_{i}^{n}, u_{i+1}^{n}\right)\right) d x-\mathbf{U}\left(u_{i+1 / 2}^{n+1, R}\right) \\
& \quad=\frac{1}{h_{x}} \int_{0}^{h_{x} / 2}\left|w\left(\cdot ; u_{i}^{n}, u_{i+1}^{n}\right)-u_{i+1 / 2}^{n+R}\right|^{2} d x,
\end{aligned}
$$

which, used in (3.10), yields the required equality (3.8a).

It is easy to verify that the terms of the right-hand sides of formulae (3.8) are nonpositive. Therefore, adding (3.8a) and (3.8b) and using (3.6a) and the property of convexity of the function $\mathbf{U}$, we obtain

$$
\mathbf{U}\left(u_{i}^{n+1}\right)-\mathbf{U}\left(u_{i}^{n}\right)+\lambda_{x}\left(F_{G, i+1 / 2}^{n}-F_{G, i-1 / 2}^{n}\right) \leq 0, \quad n \in \mathbb{N}, i \in \mathbb{Z} .
$$

Inequality (3.12) is the so-called entropy inequality satisfied by the Godunov scheme (see also, for instance, Crandall and Majda [8]).

In this work, we are interested in a stronger version of this type of inequality. Namely, we want to estimate carefully the right-hand sides of (3.8) in order to derive a "sharp" entropy inequality. For this purpose, we notice that Lemma 3.1 shows that both the shock waves of the solutions of Riemann problems and the $L^{2}$-type projections used in the Godunov method generate some entropy dissipation. We expect to estimate these terms of entropy dissipation in terms of the wave magnitude $\left|u_{i+1}^{n}-u_{i}^{n}\right|$ of each Riemann problem. For each $n$ in $\mathbb{N}$ and $i$ in $\mathbb{Z}$, we shall distinguish between two cases, depending on whether $w\left(\cdot ; u_{i}^{n}, u_{i+1}^{n}\right)$ is a shock wave or a rarefaction wave $(\delta$ below is defined by (3.1)).

Lemma 3.2 (Entropy dissipation of a shock wave in the Godunov scheme). Consider the Godunov scheme under the CFL condition (3.5). Suppose that, for some $n$ in $\mathbb{N}$ and $i$ in $\mathbb{Z}$, the Riemann solution $w\left(\cdot ; u_{i}^{n}, u_{i+1}^{n}\right)$ consists of a shock wave (i.e., $\left.u_{i}^{n}>u_{i+1}^{n}\right)$ whose speed is denoted by $\sigma_{i+1 / 2}^{n}$. Then we have

$$
\begin{aligned}
& \mathbf{U}\left(u_{i+1 / 2}^{n+1, R}\right)-\mathbf{U}\left(u_{i+1}^{n}\right)+2 \lambda_{x}\left(\mathbf{F}\left(u_{i+1}^{n}\right)-F_{G, i+1 / 2}^{n}\right) \\
& \quad \leq-\lambda_{x} s_{i+1 / 2}^{n}\left\{\frac{\delta}{6}\left|u_{i+1}^{n}-u_{i}^{n}\right|^{3}+\sigma_{i+1 / 2}^{n}\left(1-2 \lambda_{x} \sigma_{i+1 / 2}^{n}\right)\left|u_{i+1}^{n}-u_{i}^{n}\right|^{2}\right\} \\
& \quad \leq 0
\end{aligned}
$$


and

$$
\begin{aligned}
\mathbf{U}\left(u_{i+1 / 2}^{n+1, L}\right)-\mathbf{U}\left(u_{i}^{n}\right)+2 \lambda_{x}\left(F_{G, i+1 / 2}^{n}-\mathbf{F}\left(u_{i}^{n}\right)\right) \\
\leq-\lambda_{x}\left(1-s_{i+1 / 2}^{n}\right)\left\{\frac{\delta}{6}\left|u_{i+1}^{n}-u_{i}^{n}\right|^{3}\right. \\
\left.\quad-\sigma_{i+1 / 2}^{n}\left(1+2 \lambda_{x} \sigma_{i+1 / 2}^{n}\right)\left|u_{i+1}^{n}-u_{i}^{n}\right|^{2}\right\} \leq 0,
\end{aligned}
$$

where $s_{i+1 / 2}^{n}$ is defined by

$$
s_{i+1 / 2}^{n}= \begin{cases}1, & \text { if } \sigma_{i+1 / 2}^{n}>0 \\ 0, & \text { if } \sigma_{i+1 / 2}^{n} \leq 0\end{cases}
$$

Remark 3.2. Under the assumptions of Lemma 3.2, suppose that the sonic point $u_{*}$ lies between $u_{i+1}^{n}$ and $u_{i}^{n}$ and that

$$
\mathbf{f}\left(u_{i+1}^{n}\right)-\mathbf{f}\left(u_{i}^{n}\right)=0
$$

i.e., $u_{i}^{n}$ and $u_{i+1}^{n}$ are connected by a stationary shock; then we have

$$
\sigma_{i+1 / 2}^{n}=0, \quad s_{i+1 / 2}^{n}=0 \text {. }
$$

Thus, in that case, inequalities (3.13) become

$$
\mathbf{U}\left(u_{i+1 / 2}^{n+1, R}\right)-\mathbf{U}\left(u_{i+1}^{n}\right)+2 \lambda_{x}\left(\mathbf{F}\left(u_{i+1}^{n}\right)-F_{G, i+1 / 2}^{n}\right) \leq 0
$$

and

$$
\mathbf{U}\left(u_{i+1 / 2}^{n+1, L}\right)-\mathbf{U}\left(u_{i}^{n}\right)+2 \lambda_{x}\left(F_{G, i+1 / 2}^{n}-\mathbf{F}\left(u_{i}^{n}\right)\right) \leq-\lambda_{x} \frac{\delta}{6}\left|u_{i+1}^{n}-u_{i}^{n}\right|^{3} .
$$

Moreover, formulae (3.8) prove in fact that the above inequalities are equalities. This shows that the local entropy dissipation of the Godunov scheme may admit a uniform cubic estimate of the form $C\left|u_{i+1}^{n}-u_{i}^{n}\right|^{3}$, but not a quadratic one. This is actually related to the property that the numerical viscosity coefficient of the scheme under consideration vanishes in the "neighborhood" of the sonic point $(\S 3.5$ treats precisely the schemes whose viscosity coefficient is uniformly bounded below; for these schemes, a quadratic rate is obtained).

Proof of Lemma 3.2. We only demonstrate (3.13a); the proof of $(3.13 \mathrm{~b})$ is similar. By Lemma 3.1 we have to estimate each term of the right-hand side of (3.8a). Note that the term $J_{i+1 / 2}^{n, R}$ defined in (3.9a) is zero when $\sigma_{i+1 / 2}^{n}$ is nonpositive, i.e., when $s_{i+1 / 2}^{n}=0$. Otherwise, we can write

$$
J_{i+1 / 2}^{n, R}=\int_{u_{i}^{n}}^{u_{i+1}^{n}} v \mathbf{f}^{\prime}(v) d v-\sigma_{i+1 / 2}^{n} \frac{1}{2}\left(\left(u_{i+1}^{n}\right)^{2}-\left(u_{i}^{n}\right)^{2}\right) .
$$

And with the Rankine-Hugoniot relation,

$$
\sigma_{i+1 / 2}^{n}\left(u_{i+1}^{n}-u_{i}^{n}\right)=\mathbf{f}\left(u_{i+1}^{n}\right)-\mathbf{f}\left(u_{i}^{n}\right),
$$


we obtain

$$
J_{i+1 / 2}^{n, R}=\int_{u_{i}^{n}}^{u_{i+1}^{n}} v \mathbf{f}^{\prime}(v) d v-\left(\mathbf{f}\left(u_{i+1}^{n}\right)-\mathbf{f}\left(u_{i}^{n}\right)\right) \frac{1}{2}\left(u_{i+1}^{n}+u_{i}^{n}\right) .
$$

By integration by parts it is easy to deduce from the above formula that

$$
J_{i+1 / 2}^{n, R}=\frac{1}{2} \int_{u_{i}^{n}}^{u_{i+1}^{n}}\left(u_{i}^{n}-v\right)\left(v-u_{i+1}^{n}\right) \mathbf{f}^{\prime \prime}(v) d v .
$$

Hence, $\delta$ being given by (3.1), we get

$$
J_{i+1 / 2}^{n, R} \leq \frac{\delta}{2} \int_{u_{i}^{n}}^{u_{i+1}^{n}}\left(u_{i}^{n}-v\right)\left(v-u_{i+1}^{n}\right) d v=-\frac{\delta}{12}\left|u_{i+1}^{n}-u_{i}^{n}\right|^{3},
$$

since $u_{i}^{n}>u_{i+1}^{n}$. We next turn to the second term of the the right-hand side of (3.8a). Since $w\left(\cdot ; u_{i}^{n}, u_{i+1}^{n}\right)$ contains a shock wave of speed $\sigma_{i+1 / 2}^{n}$, which can be positive or not, we set $\sigma_{i+1 / 2,+}^{n}=\max \left(0, \sigma_{i+1 / 2}^{n}\right)$ and get

$$
\begin{aligned}
& \frac{1}{h_{x}} \int_{0}^{h_{x} / 2} \mid w\left(\left(\frac{x}{h} ; u_{i}^{n}, u_{i+1}^{n}\right)-\left.u_{i+1 / 2}^{n+1, R}\right|^{2} d x\right. \\
& \quad=\lambda_{x} \sigma_{i+1 / 2,+}^{n}\left(u_{i}^{n}-u_{i+1 / 2}^{n+1, R}\right)^{2}+\frac{\left(1-2 \lambda_{x} \sigma_{i+1 / 2,+}^{n}\right)}{2}\left(u_{i+1}^{n}-u_{i+1 / 2}^{n+1, R}\right)^{2} .
\end{aligned}
$$

On the other hand, (3.6b) yields

$$
u_{i+1 / 2}^{n+1, R}=2 \lambda_{x} \sigma_{i+1 / 2,+}^{n} u_{i}^{n}+\left(1-2 \lambda_{x} \sigma_{i+1 / 2,+}^{n}\right) u_{i+1}^{n} ;
$$

thus,

$$
u_{i}^{n}-u_{i+1 / 2}^{n+1, R}=\left(1-2 \lambda_{x} \sigma_{i+1 / 2,+}^{n}\right)\left(u_{i}^{n}-u_{i+1}^{n}\right)
$$

and

$$
u_{i+1}^{n}-u_{i+1 / 2}^{n+1, R}=2 \lambda_{x} \sigma_{i+1 / 2,+}^{n}\left(u_{i+1}^{n}-u_{i}^{n}\right) .
$$

Using (3.18) in (3.17), we finally find

$$
\begin{aligned}
& \frac{1}{h_{x}} \int_{0}^{h_{x} / 2}\left|w\left(\frac{x}{h} ; u_{i}^{n}, u_{i+1}^{n}\right)-u_{i+1 / 2}^{n+1, R}\right|^{2} d x \\
& \quad=\lambda_{x} \sigma_{i+1 / 2,+}^{n}\left(1-2 \lambda_{x} \sigma_{i+1 / 2,+}^{n}\right)\left(u_{i}^{n}-u_{i+1}^{n}\right)^{2} .
\end{aligned}
$$

The conclusion follows immediately from (3.8a), (3.16), and (3.19).

Our second result treats rarefaction waves $\left(u_{*}\right.$ below is the sonic point defined by $(3.2))$.

Lemma 3.3 (Entropy dissipation of a rarefaction wave in the Godunov scheme). Consider the Godunov scheme under the CFL condition (3.5). Suppose that, for some $n$ in $\mathbb{N}$ and $i$ in $\mathbb{Z}$, the Riemann solution $w\left(\cdot ; u_{i}^{n}, u_{i+1}^{n}\right)$ consists of $a$ rarefaction wave (i.e., $\left.u_{i}^{n}<u_{i+1}^{n}\right)$. Then, setting

$$
\sigma_{i+1 / 2}^{n, L}=\mathbf{f}^{\prime}\left(u_{i}^{n}\right), \quad \sigma_{i+1 / 2}^{n}=\frac{\mathbf{f}\left(u_{i+1}^{n}\right)-\mathbf{f}\left(u_{i}^{n}\right)}{u_{i+1}^{n}-u_{i}^{n}}, \quad \sigma_{i+1 / 2}^{n, R}=\mathbf{f}^{\prime}\left(u_{i+1}^{n}\right),
$$


we have

$$
\begin{aligned}
& \mathbf{U}\left(u_{i+1 / 2}^{n+1, R}\right)-\mathbf{U}\left(u_{i+1}^{n}\right)+2 \lambda_{x}\left(\mathbf{F}\left(u_{i+1}^{n}\right)-F_{G, i+1 / 2}^{n}\right) \\
& \leq-\lambda_{x} s_{i+1 / 2}^{n, R}\left\{\frac{\delta}{12}\left(\left(1-s_{i+1 / 2}^{n, L}\right)\left|u_{i+1}^{n}-u_{*}\right|^{3}+s_{i+1 / 2}^{n, L}\left|u_{i+1}^{n}-u_{i}^{n}\right|^{3}\right)\right\} \\
& \quad-\lambda_{x} s_{i+1 / 2}^{n, R}\left\{s_{i+1 / 2}^{n, L} \sigma_{i+1 / 2}^{n, L}\left(1-2 \lambda_{x} \sigma_{i+1 / 2}^{n}\right)^{2}\right. \\
& \left.\quad+2 \lambda_{x}\left(1-2 \lambda_{x} \sigma_{i+1 / 2}^{n, R}\right)\left(\sigma_{i+1 / 2}^{n}\right)^{2}\right\}\left|u_{i+1}^{n}-u_{i}^{n}\right|^{2} \leq 0
\end{aligned}
$$

and

$$
\begin{aligned}
& \mathbf{U}\left(u_{i+1 / 2}^{n+1, L}\right)-\mathbf{U}\left(u_{i}^{n}\right)+2 \lambda_{x}\left(F_{G, i+1 / 2}^{n}-\mathbf{F}\left(u_{i}^{n}\right)\right) \\
& \leq-\lambda_{x}\left(1-s_{i+1 / 2}^{n, L}\right) \frac{\delta}{12}\left\{s_{i+1 / 2}^{n, R}\left|u_{*}-u_{i}^{n}\right|^{3}+\left(1-s_{i+1 / 2}^{n, R}\right)\left|u_{i+1}^{n}-u_{i}^{n}\right|^{3}\right\} \\
& -\lambda_{x}\left(1-s_{i+1 / 2}^{n, L}\right) \\
& \quad\left\{\left(1+2 \lambda_{x} \sigma_{i+1 / 2}^{n, L}\right) 2 \lambda_{x}\left(\sigma_{i+1 / 2}^{n}\right)^{2}\right. \\
& \left.-\left(1-s_{i+1 / 2}^{n, R}\right) \sigma_{i+1 / 2}^{n, R}\left(1+2 \lambda_{x} \sigma_{i+1 / 2}^{n}\right)^{2}\right\}\left|u_{i+1}^{n}-u_{i}^{n}\right|^{2} \leq 0,
\end{aligned}
$$

where $s_{i+1 / 2}^{n, L}$ and $s_{i+1 / 2}^{n, R}$ are defined by $(\alpha=L$ or $R)$

$$
s_{i+1 / 2}^{n, \alpha}= \begin{cases}1, & \text { if } \sigma_{i+1 / 2}^{n, \alpha}>0 \\ 0, & \text { if } \sigma_{i+1 / 2}^{n, \alpha} \leq 0\end{cases}
$$

Remark 3.3. Under the assumption of Lemma 3.3, if $u_{*}$ lies between $u_{i}^{n}$ and $u_{i+1}^{n}$ and the relation $\mathbf{f}\left(u_{i+1}^{n}\right)-\mathbf{f}\left(u_{i}^{n}\right)=0$ holds, then

$$
s_{i+1 / 2}^{n, L}=0, \quad s_{i+1 / 2}^{n, R}=1, \quad \sigma_{i+1 / 2}^{n}=0
$$

Thus, in that case, inequalities $(3.21)$ become

$$
\mathbf{U}\left(u_{i+1 / 2}^{n+1, R}\right)-\mathbf{U}\left(u_{i+1}^{n}\right)+2 \lambda_{x}\left(\mathbf{F}\left(u_{i+1}^{n}\right)-F_{G, i+1 / 2}^{n}\right) \leq-\lambda_{x} \frac{\delta}{12}\left|u_{i+1}^{n}-u_{*}\right|^{3}
$$

and

$$
\mathbf{U}\left(u_{i+1 / 2}^{n+1, L}\right)-\mathbf{U}\left(u_{i}^{n}\right)+2 \lambda_{x}\left(F_{G, i+1 / 2}^{n}-\mathbf{F}\left(u_{i}^{n}\right)\right) \leq-\lambda_{x} \frac{\delta}{12}\left|u_{*}-u_{i}^{n}\right|^{3}
$$

This shows again that no uniform quadratic estimate can be obtained in that case, but only a cubic estimate.

Proof of Lemma 3.3. We give the proof of (3.21a). By Lemma 3.1, the entropy dissipation in that case is only due to the error in the $L^{2}$ projection 
step (see $(3.6 \mathrm{~b}),(3.6 \mathrm{c}))$. Setting $\sigma_{i+1 / 2,+}^{n, L}=\max \left(0, \sigma_{i+1 / 2}^{n, L}\right)$ and $\sigma_{i+1 / 2,+}^{n, R}=$ $\max \left(0, \sigma_{i+1 / 2}^{n, R}\right)$, we have

$$
\begin{aligned}
& \frac{1}{h_{x}} \int_{0}^{h_{x} / 2}\left|w\left(\frac{x}{h} ; u_{i}^{n}, u_{i+1}^{n}\right)-u_{i+1 / 2}^{n+1, R}\right|^{2} d x \\
& =\lambda_{x} \sigma_{i+1 / 2,+}^{n, L}\left(u_{i}^{n}-u_{i+1 / 2}^{n+1, R}\right)^{2}+\frac{\left(1-2 \lambda_{x} \sigma_{i+1 / 2,+}^{n, R}\right)}{2}\left(u_{i+1}^{n}-u_{i+1 / 2}^{n+1, R}\right)^{2} \\
& \quad+\frac{1}{h_{x}} \int_{h \sigma_{i+1 / 2,+}^{n, L}}^{h \sigma_{i+1 / 2,+}^{n, R}}\left|w\left(\frac{x}{h} ; u_{i}^{n}, u_{i+1}^{n}\right)-u_{i+1 / 2}^{n+1, R}\right|^{2} d x
\end{aligned}
$$

The first two terms of the right-hand side of (3.24), denoted by $R_{1}$, can be estimated as follows. By (3.6b), we have

$$
\begin{aligned}
u_{i+1 / 2}^{n+1, R}= & 2 \lambda_{x} \sigma_{i+1 / 2,+}^{n, L} u_{i}^{n}+\left(1-2 \lambda_{x} \sigma_{i+1 / 2,+}^{n, R}\right) u_{i+1}^{n} \\
& +\frac{2}{h_{x}} \int_{h \sigma_{i+1 / 2,+}^{n, L}}^{h \sigma_{i+1 / 2,+}^{n, R}} w\left(\frac{x}{h} ; u_{i}^{n}, u_{i+1}^{n}\right) d x .
\end{aligned}
$$

Since $w\left(\cdot ; u_{i}^{n}, u_{i+1}^{n}\right)$ is a rarefaction wave, we have

$$
w\left(\xi ; u_{i}^{n}, u_{i+1}^{n}\right)=\left(\mathbf{f}^{\prime}\right)^{-1}(\xi) \quad \text { for } \xi \in\left[\mathbf{f}^{\prime}\left(u_{i}^{n}\right), \mathbf{f}^{\prime}\left(u_{i+1}^{n}\right)\right]
$$

Hence, by a change of variables, we can get

$$
\frac{2}{h_{x}} \int_{h \sigma_{i+1 / 2,+}^{n, L}}^{h \sigma_{i+1 / 2,+}^{n, R}} w\left(\frac{x}{h} ; u_{i}^{n}, u_{i+1}^{n}\right) d x=2 \lambda_{x} \int_{u_{i,+}^{n}}^{u_{i+1,+}^{n}} u \mathbf{f}^{\prime \prime}(u) d u
$$

where by convention we set

$$
u_{j,+}^{n}=\max \left(u_{*}, u_{j}^{n}\right), \quad j \in \mathbb{Z},
$$

$u_{*}$ being given by (3.2). Thus, we have after an easy computation

$$
\begin{aligned}
& \frac{2}{h_{x}} \int_{h \sigma_{i+1 / 2,+}^{n, L}}^{h \sigma_{i+1 / 2,+}^{n, R}} w\left(\frac{x}{h} ; u_{i}^{n}, u_{i+1}^{n}\right) d x \\
& \quad=2 \lambda_{x}\left\{u_{i+1,+}^{n} \mathbf{f}^{\prime}\left(u_{i+1,+}^{n}\right)-u_{i,+}^{n} \mathbf{f}^{\prime}\left(u_{i,+}^{n}\right)-\mathbf{f}\left(u_{i+1,+}^{n}\right)+\mathbf{f}\left(u_{i,+}^{n}\right)\right\},
\end{aligned}
$$

that is

$$
\begin{aligned}
& \frac{2}{h_{x}} \int_{h \sigma_{i+1 / 2,+}^{n, L}}^{h \sigma_{i+1 / 2,+}^{n, R}} w\left(\frac{x}{h} ; u_{i}^{n}, u_{i+1}^{n}\right) d x \\
& \quad=2 \lambda_{x}\left\{\sigma_{i+1 / 2,+}^{n, R} u_{i+1}^{n}-\sigma_{i+1 / 2,+}^{n, L} u_{i}^{n}-\mathbf{f}\left(u_{i+1,+}^{n}\right)+\mathbf{f}\left(u_{i,+}^{n}\right)\right\}
\end{aligned}
$$

Using (3.27) in (3.25), we find the formula

$$
u_{i+1 / 2}^{n+1, R}=u_{i+1}^{n}-2 \lambda_{x}\left(\mathbf{f}\left(u_{i+1,+}^{n}\right)-\mathbf{f}\left(u_{i,+}^{n}\right)\right),
$$


from which we deduce

$$
\left(u_{i}^{n}-u_{i+1 / 2}^{n+1, R}\right)=\left(u_{i}^{n}-u_{i+1}^{n}\right)+2 \lambda_{x}\left(\mathbf{f}\left(u_{i+1,+}^{n}\right)-\mathbf{f}\left(u_{i,+}^{n}\right)\right)
$$

and

$$
\left(u_{i+1}^{n}-u_{i+1 / 2}^{n+1, R}\right)=2 \lambda_{x}\left(\mathbf{f}\left(u_{i+1,+}^{n}\right)-\mathbf{f}\left(u_{i,+}^{n}\right)\right) .
$$

Finally, with obvious notation, the first two terms in the right-hand side of (3.24) become, because of (3.29),

$$
\begin{aligned}
R_{1} \equiv \lambda_{x} \sigma_{i+1 / 2,+}^{n, L}\left(u_{i}^{n}-u_{i+1 / 2}^{n+1, R}\right)^{2}+\frac{\left(1-2 \lambda_{x} \sigma_{i+1 / 2,+}^{n, R}\right)}{2}\left(u_{i+1}^{n}-u_{i+1 / 2}^{n+1, R}\right)^{2} \\
=\lambda_{x}\left\{\mathbf{f}^{\prime}\left(u_{i,+}^{n}\right)\left(1-2 \lambda_{x} \frac{\mathbf{f}\left(u_{i+1,+}^{n}\right)-\mathbf{f}\left(u_{i,+}^{n}\right)}{u_{i+1}^{n}-u_{i}^{n}}\right)^{2}\right. \\
\left.\quad+2 \lambda_{x}\left(1-2 \lambda_{x} \mathbf{f}^{\prime}\left(u_{i+1,+}\right)\right)\left(\frac{\mathbf{f}\left(u_{i+1,+}^{n}-\mathbf{f}\left(u_{i,+}^{n}\right)\right.}{u_{i+1}^{n}-u_{i}^{n}}\right)^{2}\right\}\left(u_{i+1}^{n}-u_{i}^{n}\right)^{2} .
\end{aligned}
$$

But since

$$
\frac{\mathbf{f}\left(u_{i+1,+}^{n}\right)-\mathbf{f}\left(u_{i,+}^{n}\right)}{u_{i+1}^{n}-u_{i}^{n}} \geq s_{i+1 / 2}^{n, R} \frac{\mathbf{f}\left(u_{i+1}^{n}\right)-\mathbf{f}\left(u_{i}^{n}\right)}{u_{i+1}^{n}-u_{i}^{n}}
$$

we obtain the estimate

$$
\begin{aligned}
R_{1} \geq \lambda_{x}\{ & s_{i+1 / 2}^{n, L} \sigma_{i+1 / 2}^{n, L}\left(1-2 \lambda_{x} \sigma_{i+1 / 2}^{n}\right)^{2} \\
& \left.+s_{i+1 / 2}^{n, R} 2 \lambda_{x}\left(1-2 \lambda_{x} \sigma_{i+1 / 2}^{n, R}\right)\left(\sigma_{i+1 / 2}^{n}\right)^{2}\right\}\left(u_{i+1}^{n}-u_{i}^{n}\right)^{2} .
\end{aligned}
$$

On the other hand, the last term, denoted by $R_{2}$, of the right-hand side of (3.24) is estimated as follows. Similarly as above, we can verify that

$$
\begin{aligned}
R_{2} & \equiv \frac{1}{h_{x}} \int_{h \sigma_{i+1 / 2,+}^{n, L}}^{h \sigma_{i+1 / 2,+}^{n, R}}\left|w\left(\frac{x}{h} ; u_{i}^{n}, u_{i+1}^{n}\right)-u_{i+1 / 2}^{n+1, R}\right|^{2} d x \\
& =\lambda_{x} \int_{u_{i,+}^{n}}^{u_{i+1,+}^{n}}\left|v-u_{i+1 / 2}^{n+1, R}\right|^{2} \mathbf{f}^{\prime \prime}(v) d v .
\end{aligned}
$$

Since $u_{i,+}^{n} \leq u_{i+1,+}^{n}$, we have

$R_{2} \geq \delta \lambda_{x} \int_{u_{i,+}^{n}}^{u_{i+1,+}^{n}}\left|v-u_{i+1 / 2}^{n+1, R}\right|^{2} d v=\frac{\delta \lambda_{x}}{3}\left\{\left(u_{i+1,+}^{n}-u_{i+1 / 2}^{n+1, R}\right)^{3}-\left(u_{i,+}^{n}-u_{i+1 / 2}^{n+1, R}\right)^{3}\right\}$,

$\delta$ being given by $(3.1)$, so that

$$
R_{2} \geq \frac{\delta \lambda_{x}}{3}\left\{\left(u_{i+1,+}^{n}-u_{i+1 / 2}^{n+1, R}\right)^{3}+\left(\left(u_{i+1,+}^{n}-u_{i,+}^{n}\right)-\left(u_{i+1,+}^{n}-u_{i+1 / 2}^{n+1, R}\right)\right)^{3}\right\} .
$$

Since for each $z_{0} \geq 0$,

$$
\min _{0 \leq z \leq z_{0}}\left(z^{3}+\left(z_{0}-z\right)^{3}\right)=z_{0}^{3} / 4
$$


it is easy to deduce from the above inequality that

$$
R_{2} \geq\left\{\begin{array}{ll}
0, & \text { if } u_{i}^{n} \leq u_{i+1}^{n} \leq u_{*}, \\
\lambda_{x} \frac{\delta}{12}\left|u_{i+1}^{n}-u_{*}\right|^{3}, & \text { if } u_{i}^{n} \leq u_{*} \leq u_{i+1}^{n}, \\
\lambda_{x} \frac{\delta}{12}\left|u_{i+1}^{n}-u_{i}^{n}\right|^{3}, & \text { if } u_{*} \leq u_{i}^{n} \leq u_{i+1}^{n}
\end{array} .\right.
$$

The required inequality (3.21a) follows immediately from (3.24), (3.30), and (3.31).

From the results of Lemmas 3.2 and 3.3, and Remarks 3.2 and 3.3, we can make the following observation concerning the Godunov scheme. The entropy dissipation of the Godunov scheme is composed of two terms. One is proportional to the modulus of convexity of the function $f$ and, roughly speaking, corresponds to the dissipation of entropy inside a shock wave or inside a rarefaction wave. This term yields below a cubic uniform estimate. The second one is generated (essentially) by the $L^{2}$ projection error. It is proportional to the speed of the waves $\left(\sigma_{i+1 / 2}^{n}\right.$ or $\left.\sigma_{i+1 / 2}^{n, L}, \sigma_{i+1 / 2}^{n, R}\right)$, which can vanish in the "neighborhood" of the sonic point $u_{*}$. This is the reason why a quadratic uniform estimate of the local entropy dissipation does not hold true for the Godunov scheme.

Hence, the "better" estimate of the entropy dissipation of the Godunov scheme is given by the following theorem.

Theorem 3.1 (A sharp entropy inequality for the Godunov scheme). Under the CFL condition (3.5), the Godunov scheme (3.6) satisfies for each integer $n$ in $\mathbb{N}$

$$
\sum_{i \in \mathbb{Z}} \mathbf{U}\left(u_{i}^{n+1}\right) h_{x}-\sum_{i \in \mathbb{Z}} \mathbf{U}\left(u_{i}^{n}\right) h_{x}+\lambda_{x} \frac{\delta}{96} \sum_{i \in \mathbb{Z}}\left|u_{i+1}^{n}-u_{i}^{n}\right|^{3} h_{x} \leq 0 .
$$

Remarks 3.4. (1) Inequality (3.32) indeed yields a uniform estimate in $L^{2}$-norm and a weak estimate of the space derivative of the form (2.15) (see $\S 4$; the initial data will be assumed there to have compact support).

(2) Theorem 3.1 (and actually all the results of this section) could be generalized to arbitrary strictly convex entropies $\mathbf{U}$; the only modification is that $\delta$ should be replaced by $\delta \min \mathbf{U}^{\prime \prime}$. But we emphasize that an inequality associated with an arbitrary entropy provides the same estimate for the space derivatives as is provided by (3.32).

(3) If the flux function $\mathbf{f}$ is not assumed to be convex, the above derivation cannot provide an estimate of the space derivatives. However, if $f^{\prime}$ is a positive function (resp. a negative function), a uniform quadratic estimate is easily deduced from results of Lemmas 3.2 and 3.3.

Proof of Theorem 3.1. By (3.6a), we have for $n$ in $\mathbb{N}$ and $i$ in $\mathbb{Z}$,

$$
\mathbf{U}\left(u_{i}^{n+1}\right) \leq \frac{1}{2}\left(\mathbf{U}\left(u_{i-1 / 2}^{n+1, R}\right)+\mathbf{U}\left(u_{i+1 / 2}^{n+1, L}\right)\right) .
$$


Using (3.13) and (3.21) and summing with respect to $i$, we obtain with obvious notation

$$
\begin{aligned}
\sum_{i \in \mathbb{Z}}\left\{\mathbf{U}\left(u_{i}^{n+1}\right)-\mathbf{U}\left(u_{i}^{n}\right)+\lambda_{x}\left(F_{G, i+1 / 2}^{n}-F_{G, i-1 / 2}^{n}\right)\right\} \\
\leq-\lambda_{x} \sum_{(i-1 / 2) \text { shock }} s_{i-1 / 2}^{n} \frac{\delta}{12}\left|u_{i}^{n}-u_{i-1}^{n}\right|^{3} \\
\quad-\lambda_{x} \sum_{(i-1 / 2) \text { rarefaction }} s_{i-1 / 2}^{n, R} \frac{\delta}{24}\left\{\left(1-s_{i-1 / 2}^{n, L}\right)\left|u_{i}^{n}-u_{*}\right|^{3}+s_{i-1 / 2}^{n, L}\left|u_{i}^{n}-u_{i-1}^{n}\right|^{3}\right\} \\
\quad-\lambda_{x} \sum_{(i+1 / 2) \text { shock }}\left(1-s_{i+1 / 2}^{n}\right) \frac{\delta}{12}\left|u_{i+1}^{n}-u_{i}^{n}\right|^{3} \\
\quad-\lambda_{x} \sum_{(i+1 / 2) \text { rarefaction }}\left(1-s_{i+1 / 2}^{n, L}\right) \frac{\delta}{24}\left\{s_{i+1 / 2}^{n, R}\left|u_{*}-u_{i}^{n}\right|^{3}\right.
\end{aligned}
$$

where we have only kept the cubic terms. So, we have

$$
\begin{aligned}
& \sum_{i \in \mathbb{Z}} \mathbf{U}\left(u_{i}^{n+1}\right)-\sum_{i \in \mathbb{Z}} \mathbf{U}\left(u_{i}^{n}\right) \\
& \leq-\frac{\lambda_{x}}{12} \sum_{(i+1 / 2) \text { shock }}\left|u_{i+1}^{n}-u_{i}^{n}\right|^{3} \\
& \quad-\lambda_{x} \frac{\delta}{24} \sum_{(i+1 / 2) \text { rarefaction }}\left\{\left(1-s_{i+1 / 2}^{n, L}\right) s_{i+1 / 2}^{n, R}\left(\left|u_{*}-u_{i}^{n}\right|^{3}+\left|u_{i+1}^{n}-u_{*}\right|^{3}\right)\right\} \\
& \quad+\left\{\left(s_{i+1 / 2}^{n, L} s_{i+1 / 2}^{n, R}+\left(1-s_{i+1 / 2}^{n, L}\right)\left(1-s_{i+1 / 2}^{n, R}\right)\right)\left|u_{i+1}^{n}-u_{i}^{n}\right|^{3}\right\} .
\end{aligned}
$$

We shall denote below by $D_{i+1 / 2}^{n}$ the term within curled parentheses in the second sum of the right-hand side of (3.33). When $w\left(\cdot ; u_{i+1}, u_{i}\right)$ for some $i$ in $\mathbb{Z}$ contains a rarefaction wave with a sonic point, that is, $s_{i+1 / 2}^{n, L}=0$ and $s_{i+1 / 2}^{n, R}=1$, we estimate $D_{i+1 / 2}^{n}$ by

$$
D_{i+1 / 2}^{n}=\left|u_{*}-u_{i}^{n}\right|^{3}+\left|u_{i+1}^{n}-u_{*}\right|^{3} \geq \frac{1}{4}\left|u_{i+1}^{n}-u_{i}^{n}\right|^{3},
$$

since $u_{i}^{n} \leq u_{*} \leq u_{i+1}^{n}$. Thus, (3.33) is equivalent to

$$
\begin{aligned}
\sum_{i \in \mathbb{Z}} \mathbf{U}\left(u_{i}^{n+1}\right)-\sum_{i \in \mathbb{Z}} \mathbf{U}\left(u_{i}^{n}\right) \leq & -\lambda_{x} \frac{\delta}{12} \sum_{(i+1 / 2) \text { shock }}\left|u_{i+1}^{n}-u_{i}^{n}\right|^{3} \\
& -\lambda_{x} \frac{\delta}{96} \sum_{(i+1 / 2) \text { rarefaction }}\left|u_{i+1}^{n}-u_{i}^{n}\right|^{3},
\end{aligned}
$$

which gives the conclusion.

3.3. A quadratic estimate of the entropy dissipation in the modified Lax-Friedrichs scheme. We turn to the derivation of the sharp entropy inequality satisfied by 
the modified Lax-Friedrichs scheme. By using the same techniques as before, we get here a uniform quadratic estimate (instead of only a cubic one for the Godunov scheme) of the rate of entropy dissipation in this scheme. The formulae below are very close to, but actually different from, the ones derived in §3.2. However, the proofs are similar and are therefore omitted. With the same notation as before, the modified Lax-Friedrichs scheme admits the following decomposition (Tadmor [45]):

$$
u_{i}^{n+1}=\frac{1}{2}\left(u_{i-1 / 2}^{n+1}+u_{i+1 / 2}^{n+1}\right), \quad n \in \mathbb{N}, i \in \mathbb{Z},
$$

where we set

$$
u_{i+1 / 2}^{n+1}=\frac{1}{h_{x}} \int_{-h_{x} / 2}^{h_{x} / 2} w\left(\frac{x}{h} ; u_{i}^{n}, u_{i+1}^{n}\right) d x, \quad n \in \mathbb{N}, i \in \mathbb{Z} .
$$

This scheme can thus be viewed as a two-cell averaging of two (noninteracting) Riemann problems. We assume here that the CFL stability condition (3.5) is satisfied.

Let us define the numerical entropy flux of the modified Lax-Friedrichs scheme, $F_{M}$, associated with the pair $(\mathbf{U}, \mathbf{F})$, by

$$
\begin{aligned}
F_{M}\left(u_{L}, u_{R}\right)= & \frac{1}{2}\left(\mathbf{F}\left(u_{L}\right)+\mathbf{F}\left(u_{R}\right)\right) \\
& -\frac{1}{4 \lambda_{x}}\left(\mathbf{U}\left(u_{R}\right)-\mathbf{U}\left(u_{L}\right)\right), \quad u_{L}, u_{R} \in \mathbb{R} .
\end{aligned}
$$

The following result gives an exact formula for the entropy dissipation generated by the scheme.

Lemma 3.4 (Modified Lax-Friedrichs scheme). Under the CFL stability condition (3.5), the sequence $\left(u_{i}^{n}\right)_{n \in \mathbb{N}, i \in \mathbb{Z}}$ constructed by the modified Lax-Friedrichs scheme (3.35), (3.36) satisfies for $n$ in $\mathbb{N}$ and $i$ in $\mathbb{Z}$

$$
\begin{aligned}
& \mathbf{U}\left(u_{i+1 / 2}^{n+1}\right)-\frac{1}{2}\left(\mathbf{U}\left(u_{i}^{n}\right)+\mathbf{U}\left(u_{i+1}^{n}\right)\right)+\lambda_{x}\left(\mathbf{F}\left(u_{i+1}^{n}\right)-\mathbf{F}\left(u_{i}^{n}\right)\right) \\
& \quad=\lambda_{x} J_{i+1 / 2}^{n}-\frac{1}{2 h_{x}} \int_{-h_{x} / 2}^{h_{x} / 2}\left|w\left(\frac{x}{h} ; u_{i}^{n}, u_{i+1}^{n}\right)-u_{i+1 / 2}^{n+1}\right|^{2} d x .
\end{aligned}
$$

In (3.38), the term $J_{i+1 / 2}^{n}$ equals zero except when $w\left(\cdot ; u_{i}^{n}, u_{i+1}^{n}\right)$ contains a shock wave with speed $\sigma_{i+1 / 2}^{n}$; in this latter case we have

$$
J_{i+1 / 2}^{n}=\mathbf{F}\left(u_{i+1}^{n}\right)-\mathbf{F}\left(u_{i}^{n}\right)-\sigma_{i+1 / 2}^{n}\left(\mathbf{U}\left(u_{i+1}^{n}\right)-\mathbf{U}\left(u_{i}^{n}\right)\right) .
$$

Remark 3.5. The left-hand side of (3.38) can be expressed from the numerical flux $F_{M}$ in two different manners:

$$
\begin{aligned}
& \mathbf{U}\left(u_{i+1 / 2}^{n+1}\right)-\frac{1}{2}\left(\mathbf{U}\left(u_{i}^{n}\right)+\mathbf{U}\left(u_{i+1}^{n}\right)\right)+\lambda_{x}\left(\mathbf{F}\left(u_{i+1}^{n}\right)-\mathbf{F}\left(u_{i}^{n}\right)\right) \\
& \quad=\mathbf{U}\left(u_{i+1 / 2}^{n+1}\right)-\mathbf{U}\left(u_{i}^{n}\right)+2 \lambda_{x}\left(F_{M, i+1 / 2}^{n}-\mathbf{F}\left(u_{i}^{n}\right)\right) \\
& \quad=\mathbf{U}\left(u_{i+1 / 2}^{n+1}\right)-\mathbf{U}\left(u_{i+1}^{n}\right)+2 \lambda_{x}\left(\mathbf{F}\left(u_{i+1}^{n}\right)-F_{M, i+1 / 2}^{n}\right) .
\end{aligned}
$$


In particular, (3.40) is helpful to verify from (3.38) that the modified LaxFriedrichs scheme satisfies the entropy inequality

$$
\mathbf{U}\left(u_{i}^{n+1}\right)-\mathbf{U}\left(u_{i}^{n}\right)+\lambda_{x}\left(F_{M, i+1 / 2}^{n}-F_{M, i-1 / 2}^{n}\right) \leq 0, \quad n \in \mathbb{N}, i \in \mathbb{Z} .
$$

Of course, as before, we want to derive from (3.38) a stronger inequality. In order to derive a sharp entropy inequality satisfied by the modified LaxFriedrichs scheme, we first treat the case of a shock wave.

Lemma 3.5 (Entropy dissipation of a shock wave in the modified Lax-Friedrichs scheme). Consider the modified Lax-Friedrichs scheme (3.35), (3.36) under the CFL condition (3.5). Suppose that, for some $n$ in $\mathbb{N}$ and $i$ in $\mathbb{Z}$, the Riemann solution $w\left(\cdot ; u_{i}^{n}, u_{i+1}^{n}\right)$ consists of a shock wave with speed $\sigma_{i+1 / 2}^{n}$. Then we have

$$
\begin{aligned}
& \mathbf{U}\left(u_{i+1 / 2}^{n+1}\right)-\frac{1}{2}\left(\mathbf{U}\left(u_{i}^{n}\right)+\mathbf{U}\left(u_{i+1}^{n}\right)\right)+\lambda_{x}\left(\mathbf{F}\left(u_{i+1}^{n}\right)-\mathbf{F}\left(u_{i}^{n}\right)\right) \\
& \quad \leq-\lambda_{x} \frac{\delta}{12}\left|u_{i+1}^{n}-u_{i}^{n}\right|^{3}-\frac{1}{16}\left(1-4\left(\lambda_{x} \sigma_{i+1 / 2}^{n}\right)^{2}\right)\left|u_{i+1}^{n}-u_{i}^{n}\right|^{2}<0 .
\end{aligned}
$$

Remark 3.6. Inequality (3.42) can be compared with (3.13). Here, the quadratic term in the right-hand side of (3.42) never vanishes and indeed can be bounded below uniformly provided that the CFL stability (3.5) is enforced, i.e.,

$$
\lambda_{x} \sup _{u}\left|\mathbf{f}^{\prime}(u)\right| \leq \frac{1}{2}(1-\theta),
$$

with some $\theta \in(0,1)$.

Lemma 3.6 (Entropy dissipation of a rarefaction wave in the modified LaxFriedrichs scheme). Consider the modified Lax-Friedrichs scheme (3.35), (3.36) under the CFL condition (3.5). Suppose that, for some $n$ in $\mathbb{N}$ and $i$ in $\mathbb{Z}$, the Riemann solution $w\left(\cdot ; u_{i}^{n}, u_{i+1}^{n}\right)$ consists of a rarefaction wave. Then setting

$$
\sigma_{i+1 / 2}^{n, L}=\mathbf{f}^{\prime}\left(u_{i}^{n}\right), \quad \sigma_{i+1 / 2}^{n}=\frac{\mathbf{f}\left(u_{i+1}^{n}\right)-\mathbf{f}\left(u_{i}^{n}\right)}{u_{i+1}^{n}-u_{i}^{n}}, \quad \sigma_{i+1 / 2}^{n, R}=\mathbf{f}^{\prime}\left(u_{i+1}^{n}\right),
$$

we have

$$
\begin{aligned}
\mathbf{U}\left(u_{i+1 / 2}^{n+1}\right)- & \frac{1}{2}\left(\mathbf{U}\left(u_{i}^{n}\right)+\mathbf{U}\left(u_{i+1}^{n}\right)\right)+\lambda_{x}\left(\mathbf{F}\left(u_{i+1}^{n}\right)-\mathbf{F}\left(u_{i}^{n}\right)\right) \\
\leq- & \lambda_{x} \frac{\delta}{24}\left|u_{i+1}^{n}-u_{i}^{n}\right|^{3} \\
- & \frac{1}{16}\left\{\left(1+2 \lambda_{x} \sigma_{i+1 / 2}^{n, L}\right)\left(1-2 \lambda_{x} \sigma_{i+1 / 2}^{n}\right)^{2}\right. \\
& \left.\quad+\left(1-2 \lambda_{x} \sigma_{i+1 / 2}^{n, R}\right)\left(1+2 \lambda_{x} \sigma_{i+1 / 2}^{n}\right)^{2}\right\}\left|u_{i+1}^{n}-u_{i}^{n}\right|^{2}<0 .
\end{aligned}
$$

Remark 3.7. Comparing (3.44) with (3.21), we notice again that the quadratic term in the right-hand side of (3.44) does not vanish in the neighborhood of the sonic point, provided that the enforced CFL condition of Remark 3.6 is met.

Finally, from Lemmas 3.5 and 3.6, we can deduce a sharp evaluation of the entropy dissipation. 
Theorem 3.2 (A sharp entropy inequality for the modified Lax-Friedrichs scheme). Let $\theta$ belong to the interval $[0,1)(\theta$ can be equal to zero $)$. Then, under the CFL stability condition

$$
\lambda_{x} \sup _{u}\left|\mathbf{f}^{\prime}(u)\right| \leq \frac{1}{2}(1-\theta)
$$

(the supremum being taken over all $u$ under consideration), the sequence $\left(u_{i}^{n}\right)_{i \in \mathbb{Z}}$ constructed by the modified Lax-Friedrichs scheme (3.35), (3.36) satisfies the following entropy inequality for each integer $n$ in $\mathbb{N}$ :

$$
\begin{aligned}
& \sum_{i \in \mathbb{Z}} \mathbf{U}\left(u_{i}^{n+1}\right) h_{x}-\sum_{i \in \mathbb{Z}} \mathbf{U}\left(u_{i}^{n}\right) h_{x} \\
& \quad+\lambda_{x} \frac{\delta}{24} \sum_{i \in \mathbb{Z}}\left|u_{i+1}^{n}-u_{i}^{n}\right|^{3} h_{x}+\frac{\theta}{16} \sum_{i \in \mathbb{Z}}\left|u_{i+1}^{n}-u_{i}^{n}\right|^{2} h_{x} \leq 0 .
\end{aligned}
$$

If $\theta=0$ in Theorem 3.2, then (3.46) reduces to the sharp entropy inequality found for the Godunov scheme. But here, in contrast with the result found for the Godunov scheme, the modified Lax-Friedrichs scheme has a uniform quadratic rate of entropy dissipation provided that the CFL condition is enforced by (3.45). Moreover, this quadratic term, $\frac{\theta}{16} \sum_{i \in \mathbb{Z}}\left|u_{i+1}^{n}-u_{i}^{n}\right|^{2} h_{x}$, is independent of both the function $f$ and the mesh ratio $\lambda_{x}$.

Remarks 3.8. (1) Theorem 3.2 could be generalized to arbitrary strictly convex entropies U (see Remark 3.4(2)).

(2) If the flux function $\mathbf{f}$ is not assumed to be convex, then it is clear from Lemmas 3.4 to 3.6 and their proofs that (3.46) still holds true, but with $\delta=0$. In particular, under the CFL condition (3.45) with $\theta \neq 0$, a uniform estimate of the space derivatives is obtained in that case.

3.4. Entropy dissipation in the $E$-schemes. The previous results are now generalized to a general $E$-scheme. By definition, the numerical flux $p$ of an $E$-scheme satisfies the following inequality (Osher [35]):

$$
\left\{\begin{array}{l}
\left(v_{1}-v_{0}\right)\left(p\left(v_{-k+1}, \ldots, v_{0}, v_{1}, \ldots, v_{k}\right)-\mathbf{f}(w)\right) \leq 0 \\
\text { for all } v_{-k+1}, \ldots, v_{k} \text { in } \mathbb{R} \text { and for every } w \text { between } v_{0} \text { and } v_{1} .
\end{array}\right.
$$

Let us also recall that an $E$-scheme admits the following viscous formulation $[17,21]$ :

$$
\begin{aligned}
& u_{i}^{n+1}= u_{i}^{n}-\frac{\lambda_{x}}{2}\left(\mathbf{f}\left(u_{i+1}^{n}\right)-\mathbf{f}\left(u_{i-1}^{n}\right)\right) \\
&+\frac{1}{2}\left\{Q_{E, i+1 / 2}^{n}\left(u_{i+1}^{n}-u_{i}^{n}\right)-Q_{E, i-1 / 2}^{n}\left(u_{i}^{n}-u_{i-1}^{n}\right)\right\}, \\
& n \in \mathbb{N}, \quad i \in \mathbb{Z},
\end{aligned}
$$

where the numerical viscosity coefficient is defined by

$$
Q_{E, i+1 / 2}^{n}=\lambda_{x} \frac{\mathbf{f}\left(u_{i+1}^{n}\right)+\mathbf{f}\left(u_{i}^{n}\right)-2 p_{i+1 / 2}^{n}}{u_{i+1}^{n}-u_{i}^{n}}, \quad n \in \mathbb{N}, i \in \mathbb{Z} .
$$


Under the CFL condition (3.5), one can prove that

$$
Q_{G, i+1 / 2}^{n} \leq Q_{E, i+1 / 2}^{n} \leq Q_{M, i+1 / 2}^{n}=\frac{1}{2}, \quad n \in \mathbb{N}, i \in \mathbb{Z},
$$

where $Q_{G, i+1 / 2}^{n}$ and $Q_{M, i+1 / 2}^{n}$ denote the numerical viscosity coefficients of the Godunov and modified Lax-Friedrichs schemes, respectively.

Following Tadmor [45], we rewrite (3.47) in the following form $(n \in \mathbb{N}, i \in$ $\mathbb{Z})$ :

$$
u_{i}^{n+1}=\frac{1}{2}\left(u_{E, i-1 / 2}^{n+1, R}+u_{E, i+1 / 2}^{n+1, L}\right)
$$

with

$$
u_{E, i-1 / 2}^{n+1, R}=u_{i}^{n}-\lambda_{x}\left(\mathbf{f}\left(u_{i}^{n}\right)-\mathbf{f}\left(u_{i-1}^{n}\right)\right)-Q_{E, i-1 / 2}^{n}\left(u_{i}^{n}-u_{i-1}^{n}\right)
$$

and

$$
u_{E, i+1 / 2}^{n+1, L}=u_{i}^{n}-\lambda_{x}\left(\mathbf{f}\left(u_{i+1}^{n}\right)-\mathbf{f}\left(u_{i}^{n}\right)\right)+Q_{E, i+1 / 2}^{n}\left(u_{i+1}^{n}-u_{i}^{n}\right) .
$$

From Theorems 3.1 and 3.2, we can deduce an estimate of the amount of entropy dissipation in an $E$-scheme.

Theorem 3.3 (A sharp entropy inequality for $E$-schemes). Under the CFL stability condition (3.5), the sequence $\left(u_{i}^{n}\right)_{i \in \mathbb{Z}}$ constructed by the E-scheme (3.47), (3.48) satisfies for each integer $n$ in $\mathbb{N}$ the following entropy inequality:

$$
\sum_{i \in \mathbb{Z}} \mathbf{U}\left(u_{i}^{n+1}\right) h_{x}-\sum_{i \in \mathbb{Z}} \mathbf{U}\left(u_{i}^{n}\right) h_{x}+\lambda_{x} \frac{\delta}{96} \sum_{i \in \mathbb{Z}}\left|u_{i+1}^{n}-u_{i}^{n}\right|^{3} h_{x} \leq 0 .
$$

Proof. Because of inequalities (3.49), for each integer $n$ in $\mathbb{N}$ and $i$ in $\mathbb{Z}$, there exists $\chi_{i+1 / 2}^{n}$ in the interval $[0,1]$ such that

$$
Q_{E, i+1 / 2}^{n}=\chi_{i+1 / 2}^{n} Q_{G, i+1 / 2}^{n}+\left(1-\chi_{i+1 / 2}^{n}\right) Q_{M, i+1 / 2}^{n} .
$$

On the other hand, we can introduce $(n \in \mathbb{N}, i \in \mathbb{Z})$

$$
u_{G, i}^{n+1}=\frac{1}{2}\left(u_{G, i-1 / 2}^{n+1, R}+u_{G, i+1 / 2}^{n+1, L}\right) \text {, }
$$

respectively

$$
u_{M, i}^{n+1}=\frac{1}{2}\left(u_{M, i-1 / 2}^{n+1, R}+u_{M, i+1 / 2}^{n+1, L}\right),
$$

where $u_{G, i-1 / 2}^{n+1, R}$ and $u_{G, i+1 / 2}^{n+1, L}$ (resp. $u_{M, i-1 / 2}^{n+1, R}$ and $u_{M, i+1 / 2}^{n+1, L}$ ) are defined by the Godunov scheme (resp. modified Lax-Friedrichs scheme) similarly as in (3.6) (resp. (3.36)). In fact, it is easy to verify from the decomposition (3.51), (3.52) that, for instance,

$$
u_{G, i-1 / 2}^{n+1, R}=u_{i}^{n}-\lambda_{x}\left(\mathbf{f}\left(u_{i}^{n}\right)-\mathbf{f}\left(u_{i-1}^{n}\right)\right)-Q_{G, i-1 / 2}^{n}\left(u_{i}^{n}-u_{i-1}^{n}\right)
$$

and

$$
u_{M, i-1 / 2}^{n+1, R}=u_{i}^{n}-\lambda_{x}\left(\mathbf{f}\left(u_{i}^{n}\right)-\mathbf{f}\left(u_{i-1}^{n}\right)\right)-Q_{M, i-1 / 2}^{n}\left(u_{i}^{n}-u_{i-1}^{n}\right),
$$

so that by (3.54) we have

$$
u_{E, i-1 / 2}^{n+1, R}=\chi_{i-1 / 2}^{n} u_{G, i-1 / 2}^{n+1, R}+\left(1-\chi_{i-1 / 2}^{n}\right) u_{M, i-1 / 2}^{n+1, R} .
$$


In the same fashion, we get

$$
u_{E, i+1 / 2}^{n+1, L}=\chi_{i+1 / 2}^{n} u_{G, i+1 / 2}^{n+1, L}+\left(1-\chi_{i+1 / 2}^{n}\right) u_{M, i+1 / 2}^{n+1, L} .
$$

Then, (3.57) leads to the following equivalent form of the $E$-scheme (3.50)(3.52):

$$
\begin{aligned}
u_{i}^{n+1}=\frac{1}{2}( & \chi_{i-1 / 2}^{n} u_{G, i-1 / 2}^{n+1, R}+\left(1-\chi_{i-1 / 2}^{n}\right) u_{M, i-1 / 2}^{n+1, R} \\
& \left.+\chi_{i+1 / 2}^{n} u_{G, i+1 / 2}^{n+1, L}+\left(1-\chi_{i+1 / 2}^{n}\right) u_{M, i+1 / 2}^{n+1, L}\right) .
\end{aligned}
$$

By the convexity of $\mathbf{U}(u)=u^{2} / 2,(3.58)$ implies

$$
\begin{aligned}
\mathbf{U}\left(u_{i}^{n+1}\right) \leq \frac{1}{2}( & \chi_{i-1 / 2}^{n} \mathbf{U}\left(u_{G, i-1 / 2}^{n+1, R}\right)+\left(1-\chi_{i-1 / 2}^{n}\right) \mathbf{U}\left(u_{M, i-1 / 2}^{n+1, R}\right) \\
+\chi_{i+1 / 2}^{n} & \left.\mathbf{U}\left(u_{G, i+1 / 2}^{n+1, L}\right)+\left(1-\chi_{i+1 / 2}^{n}\right) \mathbf{U}\left(u_{M, i+1 / 2}^{n+1, L}\right)\right) .
\end{aligned}
$$

Summing with respect to $i$ in $\mathbb{Z}$ and using Lemmas 3.2, 3.3, 3.5, and 3.6, we obtain

$$
\begin{aligned}
& \sum_{i \in \mathbb{Z}} \mathbf{U}\left(u_{i}^{n+1}\right)-\sum_{i \in \mathbb{Z}} \mathbf{U}\left(u_{i}^{n}\right) \\
& \leq-\lambda_{x} \sum_{(i-1 / 2) \text { shock }} \chi_{i-1 / 2}^{n} s_{i-1 / 2}^{n} \frac{\delta}{12}\left|u_{i}^{n}-u_{i-1}^{n}\right|^{3} \\
& -\lambda_{x} \sum_{(i-1 / 2) \text { shock }}\left(1-\chi_{i-1 / 2}^{n}\right) \frac{\delta}{24}\left|u_{i}^{n}-u_{i-1}^{n}\right|^{3} \\
& -\lambda_{x} \sum_{(i-1 / 2) \text { rarefaction }} \chi_{i-1 / 2}^{n} s_{i-1 / 2}^{n, R} \frac{\delta}{24}\left\{\left(1-s_{i-1 / 2}^{n, L}\right)\left|u_{i}^{n}-u_{*}\right|^{3}\right. \\
& \left.+s_{i-1 / 2}^{n, L}\left|u_{i}^{n}-u_{i-1}^{n}\right|^{3}\right\} \\
& -\lambda_{x} \sum_{(i-1 / 2) \text { rarefaction }}\left(1-\chi_{i-1 / 2}^{n}\right) \frac{\delta}{48}\left|u_{i+1}^{n}-u_{i}^{n}\right|^{3} \\
& -\lambda_{x} \sum_{(i+1 / 2) \text { shock }} \chi_{i+1 / 2}^{n}\left(1-s_{i+1 / 2}^{n}\right) \frac{\delta}{12}\left|u_{i+1}^{n}-u_{i}^{n}\right|^{3} \\
& -\lambda_{x} \sum_{(i+1 / 2) \text { shock }}\left(1-\chi_{i+1 / 2}^{n}\right) \frac{\delta}{24}\left|u_{i+1}^{n}-u_{i}^{n}\right|^{3} \\
& -\lambda_{x} \sum_{(i+1 / 2)} \chi_{i+1 / 2}^{n}\left(1-s_{i+1 / 2}^{n, L}\right) \frac{\delta}{24}\left\{s_{i+1 / 2}^{n, R}\left|u_{*}-u_{i}^{n}\right|^{3}\right. \\
& \left.+\left(1-s_{i+1 / 2}^{n, R}\right)\left|u_{i+1}^{n}-u_{i}^{n}\right|^{3}\right\} \\
& -\lambda_{x} \sum_{(i+1 / 2)}\left(1-\chi_{i+1 / 2}^{n}\right) \frac{\delta}{48}\left|u_{i+1}^{n}-u_{i}^{n}\right|^{3} \text {, }
\end{aligned}
$$


the notation used here being that introduced previously. Thus, we have

$$
\begin{aligned}
\sum_{i \in \mathbb{Z}} \mathbf{U} & \left(u_{i}^{n+1}\right)-\sum_{i \in \mathbb{Z}} \mathbf{U}\left(u_{i}^{n}\right) \\
\leq & -\lambda_{x} \frac{\delta}{12} \sum_{(i+1 / 2) \text { shock }}\left(\chi_{i+1 / 2}^{n}+\left(1-\chi_{i+1 / 2}^{n}\right)\right)\left|u_{i+1}^{n}-u_{i}^{n}\right|^{3} \\
& -\lambda_{x} \frac{\delta}{96} \sum_{(i+1 / 2) \text { rarefaction }}\left(\chi_{i+1 / 2}^{n}+\left(1-\chi_{i+1 / 2}^{n}\right)\right)\left|u_{i+1}^{n}-u_{i}^{n}\right|^{3} \\
\leq & -\lambda_{x} \frac{\delta}{96} \sum_{i \in \mathbb{Z}}\left|u_{i+1}^{n}-u_{i}^{n}\right|^{3} .
\end{aligned}
$$

This completes the proof of the theorem.

3.5. A generalization. We derive now a uniform quadratic estimate of the entropy dissipation generated by the difference schemes whose viscosity coefficient is bounded below by a positive constant. This result is motivated by Theorem 3.2, which gives such a quadratic estimate for the modified Lax-Friedrichs scheme, while, as we have shown in $\S 3.2$ (Theorem 3.1), only a cubic one is possible for the Godunov scheme. This is precisely due to the fact that the numerical viscosity coefficient of the modified Lax-Friedrichs scheme is constant (equal to $1 / 2$ ) while the one of the Godunov scheme can vanish near the sonic point.

We consider here 3-point difference schemes, characterized by their numerical flux function $f: \mathbb{R}^{2} \rightarrow \mathbb{R}$, which admit the following incremental form $(n \in$ $\mathbb{N}, i \in \mathbb{Z})$

$$
u_{i}^{n+1}=u_{i}^{n}-C_{i-1 / 2}^{n}\left(u_{i}^{n}-u_{i-1}^{n}\right)+D_{i+1 / 2}^{n}\left(u_{i+1}^{n}-u_{i}^{n}\right),
$$

with as usual

$$
C_{i-1 / 2}^{n}=C\left(u_{i-1}^{n}, u_{i}^{n}\right), \quad D_{i+1 / 2}^{n}=D\left(u_{i}^{n}, u_{i+1}^{n}\right),
$$

where the Lipschitz continuous functions $C$ and $D: \mathbb{R}^{2} \rightarrow \mathbb{R}$ are given by

$$
\begin{aligned}
& C(u, v)=\lambda_{x} \frac{\mathbf{f}(v)-f(u, v)}{v-u}, \\
& D(u, v)=\lambda_{x} \frac{\mathbf{f}(u)-f(u, v)}{v-u},
\end{aligned} \quad u, v \in \mathbb{R} .
$$

The numerical viscosity coefficient $Q: \mathbb{R}^{2} \rightarrow \mathbb{R}$ is defined from $C$ and $D$ by

$$
Q(u, v)=C(u, v)+D(u, v), \quad u, v \in \mathbb{R} .
$$

Then we prove:

Theorem 3.4 (A sharp entropy inequality for a class of difference schemes). Consider the difference scheme in incremental form (3.59).

Let $\theta$ be given in the interval $(0,1)$ and assume that

$$
\begin{array}{ll}
0 \leq C(u, v), \quad 0 \leq D(u, v), & u, v \in \mathbb{R}, \\
0<K_{0} \leq Q(u, v) \leq \frac{1}{2}(1-\theta), & u, v \in \mathbb{R},
\end{array}
$$


where $K_{0}$ is a given positive constant. Then the scheme satisfies the following inequality $(n \in \mathbb{N})$ :

$$
\sum_{i \in \mathbb{Z}} U\left(u_{i}^{n+1}\right) h_{x}-\sum_{i \in \mathbb{Z}} U\left(u_{i}^{n}\right) h_{x}+\frac{K}{2} \sum_{i \in \mathbb{Z}}\left|u_{i+1}^{n}-u_{i}^{n}\right|^{2} h_{x} \leq 0,
$$

where the constant $K$ depends on $K_{0}, \lambda_{x}, \delta, \theta$, and $\sup _{i, n}\left|u_{i}^{n}\right|$ and is given explicitly by

$$
K=K_{0}-\frac{\lambda_{x}}{3} \sup _{i, n}\left(\left|f^{\prime \prime}\left(u_{i}^{n}\right) u_{i}^{n}\right|\right)-\frac{1}{2}(1-\theta)^{2} .
$$

Remarks 3.9. (1) Notice that, when $K_{0}$ is different from $\frac{1}{2}(1-\theta)$, the constant $K$ is positive for $\lambda_{x}$ small enough.

(2) The convexity of the function $f$ is not needed in Theorem 3.4.

Proof of Theorem 3.4. For $n$ in $\mathbb{N}$ and $i$ in $\mathbb{Z}$, we can write

$$
\begin{aligned}
\mathbf{U}\left(u_{i}^{n+1}\right)= & \mathbf{U}\left(u_{i}^{n}\right)+\left(u_{i}^{n+1}-u_{i}^{n}\right) u_{i}^{n}+\frac{1}{2}\left|u_{i}^{n+1}-u_{i}^{n}\right|^{2} \\
= & \mathbf{U}\left(u_{i}^{n}\right)-\lambda_{x}\left(f_{i+1 / 2}^{n}-f_{i-1 / 2}^{n}\right) u_{i}^{n}+\frac{1}{2} C_{i-1 / 2}^{n}\left(C_{i-1 / 2}^{n}+D_{i+1 / 2}^{n}\right)\left|u_{i}^{n}-u_{i-1}^{n}\right|^{2} \\
& +\frac{1}{2} D_{i+1 / 2}^{n}\left(C_{i-1 / 2}^{n}+D_{i+1 / 2}^{n}\right)\left|u_{i+1}^{n}-u_{i}^{n}\right|^{2}-\frac{1}{2} C_{i-1 / 2}^{n} D_{i+1 / 2}^{n}\left|u_{i+1}^{n}-u_{i-1}^{n}\right|^{2} .
\end{aligned}
$$

Thus, after summation with respect to $i$, we find

$$
\begin{aligned}
\sum_{i \in \mathbb{Z}} \mathbf{U}\left(u_{i}^{n+1}\right)= & \sum_{i \in \mathbb{Z}} \mathbf{U}\left(u_{i}^{n}\right)-\lambda_{x} \sum_{i \in \mathbb{Z}}\left(f_{i+1 / 2}^{n}-f_{i-1 / 2}^{n}\right) u_{i}^{n} \\
& +\frac{1}{2} \sum_{i \in \mathbb{Z}}\left(C_{i-1 / 2}^{n}+D_{i+1 / 2}^{n}\right)\left\{C_{i-1 / 2}^{n}\left|u_{i}^{n}-u_{i-1}^{n}\right|^{2}\right. \\
& \left.\quad+D_{i+1 / 2}^{n}\left|u_{i+1}^{n}-u_{i}^{n}\right|^{2}\right\} \\
& -\frac{1}{2} \sum_{i \in \mathbb{Z}} C_{i-1 / 2}^{n} D_{i+1 / 2}^{n}\left|u_{i+1}^{n}-u_{i-1}^{n}\right|^{2} .
\end{aligned}
$$

On the one hand, we notice that

$$
\sum_{i \in \mathbb{Z}}\left(f_{i+1 / 2}^{n}-f_{i-1 / 2}^{n}\right) u_{i}^{n}=-\sum_{i \in \mathbb{Z}}\left(u_{i+1}^{n}-u_{i}^{n}\right) f_{i+1 / 2}^{n}=-\sum_{i \in \mathbb{Z}} \int_{u_{i}^{n}}^{u_{i+1}^{n}} f\left(u_{i}^{n}, u_{i+1}^{n}\right) d v,
$$

thus,

$$
\sum_{i \in \mathbb{Z}}\left(f_{i+1 / 2}^{n}-f_{i-1 / 2}^{n}\right) u_{i}^{n}=-\sum_{i \in \mathbb{Z}} \int_{u_{i}^{n}}^{u_{i+1}^{n}}\left(f\left(u_{i}^{n}, u_{i+1}^{n}\right)-\mathbf{f}(v)\right) d v .
$$

And with the help of the formula

$$
\int_{a}^{b} g(v) d v=(b-a) \frac{1}{2}(g(a)+g(b))+\int_{a}^{b} \frac{1}{2}(v-a)(v-b) g^{\prime \prime}(v) d v,
$$


with $a=u_{i}^{n}, b=u_{i+1}^{n}$, and $g(v)=f\left(u_{i}^{n}, u_{i+1}^{n}\right)-\mathbf{f}(v)$, (3.67) becomes

$$
\begin{aligned}
\lambda_{x} \sum_{i \in \mathbb{Z}}\left(f_{i+1 / 2}^{n}-f_{i-1 / 2}^{n}\right) u_{i}^{n}= & \sum_{i \in \mathbb{Z}} \frac{1}{2}\left(C_{i+1 / 2}^{n}+D_{i+1 / 2}^{n}\right)\left|u_{i+1}^{n}-u_{i}^{n}\right|^{2} \\
& +\lambda_{x} \sum_{i \in \mathbb{Z}} \int_{u_{i}^{n}}^{u_{i+1}^{n}} \frac{1}{2}\left(v-u_{i}^{n}\right)\left(v-u_{i+1}^{n}\right) f^{\prime \prime}(v) d v .
\end{aligned}
$$

Hence, using (3.63), we obtain from (3.68)

$$
\begin{aligned}
-\lambda_{x} \sum_{i \in \mathbb{Z}}\left(f_{i+1 / 2}^{n}-f_{i-1 / 2}^{n}\right) u_{i}^{n} \leq & -\frac{K_{0}}{2} \sum_{i \in \mathbb{Z}}\left|u_{i+1}^{n}-u_{i}^{n}\right|^{2} \\
& +\frac{\lambda_{x}}{12}\left\|f^{\prime \prime}(u)\right\|_{L^{\infty}} \sum_{i \in \mathbb{Z}}\left|u_{i+1}^{n}-u_{i}^{n}\right|^{3} .
\end{aligned}
$$

On the other hand, we write

$$
\begin{aligned}
& \frac{1}{2} \sum_{i \in \mathbb{Z}}\left(C_{i-1 / 2}^{n}+D_{i+1 / 2}^{n}\right)\left\{C_{i-1 / 2}^{n}\left|u_{i}^{n}-u_{i-1}^{n}\right|^{2}+D_{i+1 / 2}^{n}\left|u_{i+1}^{n}-u_{i}^{n}\right|^{2}\right\} \\
& \quad-\frac{1}{2} \sum_{i \in \mathbb{Z}} C_{i-1 / 2}^{n} D_{i+1 / 2}^{n}\left|u_{i+1}^{n}-u_{i-1}^{n}\right|^{2} \leq \frac{1}{4}(1-\theta)^{2} \sum_{i \in \mathbb{Z}}\left|u_{i+1}^{n}-u_{i}^{n}\right|^{2} .
\end{aligned}
$$

Finally, combining (3.60), (3.70), and (3.66), we find

$$
\begin{aligned}
& \sum_{i \in \mathbb{Z}} \mathbf{U}\left(u_{i}^{n+1}\right)-\sum_{i \in \mathbb{Z}} \mathbf{U}\left(u_{i}^{n}\right) \\
& \quad \leq \sum_{i \in \mathbb{Z}}\left\{-\frac{K_{0}}{2}+\frac{\lambda_{x}}{12} \sup _{i, n}\left|f^{\prime \prime}\left(u_{i}^{n}\right)\right|\left|u_{i+1}^{n}-u_{i}^{n}\right|+\frac{1}{4}(1-\theta)^{2}\right\}\left|u_{i+1}^{n}-u_{i}^{n}\right|^{2},
\end{aligned}
$$

which completes the proof.

\section{THE CORRECTED ANTIDIFFUSIVE FLUX APPROACH}

4.1. Introduction. We now consider the class of high-order accurate explicit finite difference schemes which are constructed from a given $E$-scheme by the corrected antidiffusive flux method. As is well known, an $E$-scheme is at most first-order accurate (Osher [35]), and the antidiffusive flux is chosen in order to ensure both the high-order accuracy and certain formal properties of stability (e.g., Davis [10] and Sweby [42]). The results of convergence known for such schemes only concern equations with one space variable (e.g., Cahouet and Coquel [3], Leroux [30], Leroux and Quesseveur [32], Majda and Osher [33], Tadmor [46], and Vila [50]). In this section, based on the results obtained in $\S 3$, we prove the convergence of these schemes with corrected antidiffusive flux applied to an equation with an arbitrary number of space variables (Theorem 4.3 below). Moreover, even for the case of one space variable, we emphasize that our result of convergence improves the existing results in this domain (see Theorems 4.1-4.3 and Corollaries 4.1-4.2). 
We use the notation introduced in $\S 2$. Let $p$ (respectively $q$ ) be a numerical $E$-flux consistent with the exact flux function $\mathbf{f}$ (resp. g). That is, $p: \mathbb{R}^{2 k} \rightarrow \mathbb{R}$ and $q: \mathbb{R}^{2 k} \rightarrow \mathbb{R}$ are Lipschitz-continuous functions satisfying (Osher [35])

$$
\left\{\begin{array}{l}
\left(v_{1}-v_{0}\right)\left(p\left(v_{-k+1}, \ldots, v_{0}, v_{1}, \ldots, v_{k}\right)-\mathbf{f}(w)\right) \leq 0 \\
\text { for all } v_{-k+1}, \ldots, v_{k} \text { in } \mathbb{R} \text { and for every } w \text { between } v_{0} \text { and } v_{1},
\end{array}\right.
$$

and

$$
\left\{\begin{array}{l}
\left(v_{1}-v_{0}\right)\left(q\left(v_{-k+1}, \ldots, v_{0}, v_{1}, \ldots, v_{k}\right)-\mathbf{g}(w)\right) \leq 0 \\
\text { for all } v_{-k+1}, \ldots, v_{k} \text { in } \mathbb{R} \text { and for every } w \text { between } v_{0} \text { and } v_{1} .
\end{array}\right.
$$

We consider the conservative difference schemes $(n \in \mathbb{N})$

$$
u_{i, j}^{n+1}=u_{i, j}^{n}-\lambda_{x}\left(f_{i+1 / 2, j}^{n}-f_{i-1 / 2, j}^{n}\right)-\lambda_{y}\left(g_{i, j+1 / 2}^{n}-g_{i, j-1 / 2}^{n}\right), \quad i, j \in \mathbb{Z},
$$

where the numerical fluxes are given in the following form:

$$
f_{i+1 / 2, j}^{n}=p_{i+1 / 2, j}^{n}+\frac{1}{\lambda_{x}} a_{i+1 / 2, j}^{n}, \quad i, j \in \mathbb{Z},
$$

and

$$
g_{i, j+1 / 2}^{n}=q_{i, j+1 / 2}^{n}+\frac{1}{\lambda_{y}} b_{i, j+1 / 2}^{n}, \quad i, j \in \mathbb{Z} .
$$

In (4.4) and (4.5), we set

$$
p_{i+1 / 2, j}^{n}=p\left(u_{i-k+1, j}^{n}, \ldots, u_{i+k, j}^{n}\right), \quad n \in \mathbb{N}, i, j \in \mathbb{Z},
$$

and

$$
q_{i, j+1 / 2}^{n}=q\left(u_{i, j-k+1}^{n}, \ldots, u_{i, j+k}^{n}\right), \quad n \in \mathbb{N}, i, j \in \mathbb{Z} .
$$

It is known that the antidiffusive fluxes $a_{i+1 / 2, j}^{n}$ and $b_{i, j+1 / 2}^{n}$ can be chosen in such a way that this scheme is high-order accurate in space in the zones of smoothness of the solution (see an example in $\S 4.2$ constructed from the notion of flux limiter). On the other hand, without loss of accuracy we can assume, as is usual, that these antidiffusive fluxes vanish with the mesh size in the following sense: there exist $\alpha$ and $\beta$ in the interval $(0,1)$ and a constant $C>0$ such that

$$
\left|a_{i+1 / 2, j}^{n}\right| \leq C h^{\alpha}, \quad\left|b_{i, j+1 / 2}^{n}\right| \leq C h^{\beta}, \quad n \in \mathbb{N}, i, j \in \mathbb{Z} .
$$

For motivations and comments concerning these conditions, we refer to Lapidus [24], Leroux [30], Majda and Osher [33], and Vila [50] (see also Tadmor [46]). Condition (4.6) is active only in the neighborhood of the points of discontinuity of the solution, and roughly speaking is expected to be sufficient to prevent the appearance of large oscillations near the discontinuities. In all that follows, the scheme is always assumed to be consistent with equation (1.1) and the initial data to be a bounded function with compact support. The results of convergence known for these schemes concern equations in one space variable and are based on an estimate of the total variation of the approximate solutions. 
See for instance Harten [21] and Sweby [42] concerning the TVD (total variation diminishing) schemes; and Cahouet and Coquel [3], Leroux [30], and Shu [39] for the TVB (total variation bounded) approach. But as far as the authors know, there is no result in the literature on the convergence of these high-order schemes in the case of an equation with several space variables.

In this section, we prove convergence of the scheme with antidiffusion (4.3)(4.5) in the case of several space variables provided that the usual condition (4.6) is satisfied. Our proof is completely different from the one known for an equation in one space dimension: it is not based on the so-called BV-estimate, but instead makes essential use of the theory of measure-valued solutions and the uniqueness theorem due to Di Perna recalled in $\S 2$. Actually, the results of this section are also based on the sharp discrete entropy inequality for an $E$ scheme derived in the previous section. The $L^{\infty}$ stability estimate is derived in $\S 4.3$. A complete proof of convergence of the scheme (4.3)-(4.5) is given in $\S 4.4$ in the particular case of an equation in one space dimension. Finally, $\S 4.5$ treats the general case.

4.2. An example: the flux limiter approach. Following an approach due to Sweby [42] and Davis [10], we present in this subsection a particular class of antidiffusive fluxes: these antidiffusive fluxes will be constructed from a given function called a flux limiter. Define the local Courant numbers by $(n \in \mathbb{N}, i, j \in \mathbb{Z})$

$$
\sigma_{i+1 / 2, j}^{n}=\lambda_{x} \frac{f\left(u_{i+1, j}^{n}\right)-f\left(u_{i, j}^{n}\right)}{u_{i+1, j}^{n}-u_{i, j}^{n}}, \quad \tau_{i, j+1 / 2}^{n}=\lambda_{y} \frac{g\left(u_{i, j+1}^{n}\right)-g\left(u_{i, j}^{n}\right)}{u_{i, j+1}^{n}-u_{i, j}^{n}},
$$

and consider the ratios of two consecutive discrete gradients given by $(n \in$ $\mathbb{N}, i, j \in \mathbb{Z})$

and

$$
r_{i+1 / 2, j}^{n}= \begin{cases}\frac{\mathbf{f}\left(u_{i, j}^{n}\right)-\mathbf{f}\left(u_{i-1, j}^{n}\right)}{\mathbf{f}\left(u_{i+1, j}^{n}\right)-\mathbf{f}\left(u_{i, j}^{n}\right)}, & \text { if } \sigma_{i+1 / 2, j}^{n} \geq 0, \\ \frac{\mathbf{f}\left(u_{i+2, j}^{n}\right)-\mathbf{f}\left(u_{i+1, j}^{n}\right)}{\mathbf{f}\left(u_{i+1, j}^{n}\right)-\mathbf{f}\left(u_{i, j}^{n}\right)}, & \text { otherwise, }\end{cases}
$$

$$
s_{i, j+1 / 2}^{n}= \begin{cases}\frac{\mathbf{g}\left(u_{i, j}^{n}\right)-\mathbf{g}\left(u_{i, j-1}^{n}\right)}{\mathbf{g}\left(u_{i, j+1}^{n}\right)-\mathbf{g}\left(u_{i, j}^{n}\right)}, & \text { if } \tau_{i+1 / 2, j}^{n} \geq 0, \\ \frac{\mathbf{g}\left(u_{i, j+2}^{n}\right)-\mathbf{g}\left(u_{i, j+1}^{n}\right)}{\mathbf{g}\left(u_{i, j+1}^{n}\right)-\mathbf{g}\left(u_{i, j}^{n}\right)}, & \text { otherwise. }\end{cases}
$$

Let us fix two arbitrary functions $\varphi$ and $\psi: \mathbb{R} \rightarrow \mathbb{R}$, the so-called flux limiter functions, and define the antidiffusive fluxes by $(n \in \mathbb{N}, i, j \in \mathbb{Z})$

$$
\begin{aligned}
& a_{i+1 / 2, j}^{n}=\frac{1}{2}\left|\sigma_{i+1 / 2, j}^{n}\right|\left(1-\left|\sigma_{i+1 / 2, j}^{n}\right|\right) \varphi\left(r_{i+1 / 2, j}^{n}\right)\left(u_{i+1, j}^{n}-u_{i, j}^{n}\right), \\
& b_{i, j+1 / 2}^{n}=\frac{1}{2}\left|\tau_{i, j+1 / 2}^{n}\right|\left(1-\left|\tau_{i, j+1 / 2}^{n}\right|\right) \psi\left(s_{i, j+1 / 2}^{n}\right)\left(u_{i, j+1}^{n}-u_{i, j}^{n}\right) .
\end{aligned}
$$

A number of choices of the functions $\varphi$ and $\psi$ have been proposed in the literature. The choice

$$
\varphi=\psi=\varphi^{\mathrm{LW}}=\psi^{\mathrm{LW}} \equiv 1
$$


corresponds to the Lax-Wendroff scheme [29], which is second-order accurate in space and time but produces spurious oscillations in the neighborhood of points of discontinuity of the solution. On the other hand, a function like the Van Leer flux limiter [49]

$$
\varphi^{\mathrm{VL}}(r)=\psi^{\mathrm{VL}}(r)=\frac{|r|+r}{1+|r|}, \quad \text { for } r \text { in } \mathbb{R},
$$

yields a scheme which roughly speaking does not produce oscillations but is second-order accurate except at the local extrema of the solution where it is only first-order accurate. In the case of an equation with only one space variable, these schemes are convergent (e.g., Cahouet and Coquel [3]) to the entropy weak solution of the problem (1.1), (1.2) for a large class of flux limiters, for instance for Van Leer's one, provided that condition (4.6) is enforced by a slight modification of (4.7) (see (4.8a) below). We refer to [3] and [42] for the precise conditions on the flux limiter which ensure that the scheme is stable in the $L^{\infty}$. and BV-norms (in one space dimension).

The general results of this section apply to these schemes with flux limiter and show their convergence in the multidimensional case. For the sake of definiteness, let us now focus for instance on the example of the Van Leer flux limiter. Following Shu [39] and Cahouet and Coquel [3], we can introduce an improved version of the scheme which is second-order accurate at any point of regularity of the solution (i.e., including the extrema). The idea consists of replacing the Van Leer flux limiter by the Lax-Wendroff one in the neighborhood of the extrema of the solution. To be precise, the uniformly accurate version of the Van Leer scheme is defined as the scheme (4.3)-(4.5), where the antidiffusive fluxes are given by the formulas

$$
\begin{aligned}
& a_{i+1 / 2, j}^{n}=\operatorname{sgn}\left(\tilde{a}_{i+1 / 2, j}^{n}\right) \min \left(\left|\tilde{a}_{i+1 / 2, j}^{n}\right|, M_{1} h^{\alpha_{1}}\right), \\
& b_{i, j+1 / 2}^{n}=\operatorname{sgn}\left(\tilde{b}_{i, j+1 / 2}^{n}\right) \min \left(\left|\tilde{b}_{i, j+1 / 2}^{n}\right|, N_{1} h^{\beta_{1}}\right),
\end{aligned}
$$

and

$$
\begin{aligned}
& \tilde{a}_{i+1 / 2, j}^{n}=\operatorname{minmod}\left(a_{i+1 / 2, j}^{\mathrm{LW}, n}, a_{i+1 / 2, j}^{\mathrm{VL}, n}+M_{2} h^{\alpha_{2}} \operatorname{sgn}\left(a_{i+1 / 2, j}^{\mathrm{LW}, n}\right)\right), \\
& \tilde{b}_{i, j+1 / 2}^{n}=\operatorname{minmod}\left(b_{i, j+1 / 2}^{\mathrm{LW}, n}, b_{i, j+1 / 2}^{\mathrm{VL}, n}+N_{2} h^{\beta_{2}} \operatorname{sgn}\left(b_{i, j+1 / 2}^{\mathrm{LW}, n}\right)\right) .
\end{aligned}
$$

The constants $M_{i}, N_{i}$ and $\alpha_{i}, \beta_{i}$ are fixed and satisfy the conditions

$$
M_{1}, M_{2}, N_{1}, N_{2}>0, \quad \alpha_{1}, \beta_{1} \in(0,1), \quad \alpha_{2}, \beta_{2} \in[1,2] .
$$

In $(4.8 \mathrm{~b}), \operatorname{minmod}(\cdot, \cdot)$ is the function defined for $u$ and $v$ in $\mathbb{R}$ by

$$
\min \bmod (v, w)= \begin{cases}\operatorname{sgn}(v) \cdot \min (|v|,|w|), & \text { if } \operatorname{sgn} v=\operatorname{sgn} w, \\ 0, & \text { otherwise. }\end{cases}
$$

Moreover, $a_{i+1 / 2, j}^{\mathrm{LW}, n}$ and $b_{i, j+1 / 2}^{\mathrm{LW}, n}$ (respectively $a_{i+1 / 2, j}^{\mathrm{VL}, n}$ and $b_{i, j+1 / 2}^{\mathrm{VL}, n}$ ) denote the antidiffusive fluxes defined by (4.7) with $\varphi=\psi=\varphi^{\mathrm{LW}}$ (resp. $\varphi=\psi=\varphi^{\mathrm{VL}}$ ). One may show that this scheme is second-order accurate in any region, including 
the extrema, where the solution is smooth. Furthermore, in the case of only one space variable, it is shown in [3] that the scheme is stable in BV-norm and converges to the unique entropy weak solution of the problem provided that the conditions $\alpha_{1} \in(0,1)$ and $\alpha_{2}=2$ are fulfilled. But in the more general case where $\alpha_{2}$ is any number in the interval $[1,2),(4.8 \mathrm{~b})$ can be viewed as a very slight correction of the Lax-Wendroff scheme and no $B V$-estimate is known in that case, even for an equation with only one space variable.

Our results of convergence below establish the convergence of the scheme with the antidiffusion (4.8) for an equation with an arbitrary number of space variables provided that $\alpha_{1}, \beta_{1} \in\left(\frac{2}{3}, 1\right)$ and $\alpha_{2}, \beta_{2} \in[1,2]$. We point out that for any $\alpha_{1}, \beta_{1}<1$ and in a zone of smoothness of the solution we have

$$
\left|\tilde{a}_{i+1 / 2, j}^{n}\right| \leq M_{1} h^{\alpha_{1}}, \quad\left|\tilde{b}_{i, j+1 / 2}^{n}\right| \leq N_{1} h^{\beta_{1}}, \quad n \in \mathbb{N}, i, j \in \mathbb{Z},
$$

provided that $M_{1}$ and $N_{1}$ are large enough, so that (4.8a) gives

$$
a_{i+1 / 2, j}^{n}=\tilde{a}_{i+1 / 2, j}^{n}, \quad b_{i, j+1 / 2}^{n}=\tilde{b}_{i, j+1 / 2}^{n}, \quad n \in \mathbb{N}, i, j \in \mathbb{Z} .
$$

Thus, (4.8a) is active only in the reighborhood of discontinuity points of the solution.

4.3. The uniform $L^{\infty}$-estimate. The following result gives a sufficient condition to ensure that the scheme (4.3)-(4.5) is stable in $L^{\infty}$-norm.

Theorem 4.1 (Uniform $L^{\infty}$-estimate). Assume that the scheme (4.3)-(4.5) admits an incremental decomposition of the form

$$
\begin{aligned}
& u_{i, j}^{n+1}= u_{i, j}^{n}-C_{i-1 / 2, j}^{n}\left(u_{i, j}^{n}-u_{i-1, j}^{n}\right)+D_{i+1 / 2, j}^{n}\left(u_{i+1, j}^{n}-u_{i, j}^{n}\right) \\
&-E_{i, j-1 / 2}^{n}\left(u_{i, j}^{n}-u_{i, j-1}^{n}\right)+F_{i, j+1 / 2}^{n}\left(u_{i, j+1}^{n}-u_{i, j}^{n}\right)+K_{i, j}^{n}, \\
& n \in \mathbb{N}, \quad i, j \in \mathbb{Z},
\end{aligned}
$$

whose coefficients satisfy the conditions

$$
\begin{array}{ll}
C_{i+1 / 2, j}^{n} \geq 0, & D_{i+1 / 2, j}^{n} \geq 0, \\
E_{i, j+1 / 2}^{n} \geq 0, & F_{i, j+1 / 2}^{n} \geq 0,
\end{array} \quad n \in \mathbb{N}, i, j \in \mathbb{Z},
$$

the CFL-like stability condition

$$
C_{i-1 / 2, j}^{n}+D_{i+1 / 2, j}^{n}+E_{i, j-1 / 2}^{n}+F_{i, j+1 / 2}^{n} \leq 1, \quad n \in \mathbb{N}, i, j \in \mathbb{Z},
$$

and

$$
\left|K_{i, j}^{n}\right| \leq C h^{\gamma}, \quad n \in \mathbb{N}, i, j \in \mathbb{Z}, \text { with } \gamma \in[1, \infty) .
$$

Then the scheme (4.3)-(4.5) is $L^{\infty}$-stable, i.e., there exists a constant $C>0$ independent of $h, h_{x}$, and $h_{y}$ such that

$$
\left|u_{i, j}^{n}\right| \leq C, \quad n \in \mathbb{N}, i, j \in \mathbb{Z} .
$$


Proof. The incremental decomposition of the scheme can be rewritten in the form

$$
\begin{aligned}
u_{i, j}^{n+1}= & \left(1-C_{i-1 / 2, j}^{n}-D_{i+1 / 2, j}^{n}-E_{i, j-1 / 2}^{n}-F_{i, j+1 / 2}^{n}\right) u_{i, j}^{n} \\
& +C_{i-1 / 2, j}^{n} u_{i-1, j}^{n}+D_{i+1 / 2, j}^{n} u_{i+1, j}^{n}+E_{i, j-1 / 2}^{n} u_{i, j-1}^{n} \\
& +F_{i, j+1 / 2}^{n} u_{i, j+1}^{n}+K_{i, j}^{n},
\end{aligned}
$$

so that, using $(4.9 \mathrm{a})-(4.9 \mathrm{c})$, we get

$$
\begin{aligned}
\left|u_{i, j}^{n+1}\right| \leq & \left(1-C_{i-1 / 2}^{n}-D_{i+1 / 2, j}^{n}-E_{i, j-1 / 2}^{n}-F_{i, j+1 / 2}^{n}\right)\left|u_{i, j}^{n}\right| \\
& +C_{i-1 / 2, j}^{n}\left|u_{i-1, j}^{n}\right|+D_{i+1 / 2, j}^{n}\left|u_{i+1, j}^{n}\right| \\
& +E_{i, j-1 / 2}^{n}\left|u_{i, j-1}^{n}\right|+F_{i, j+1 / 2}^{n}\left|u_{i, j+1}^{n}\right|+C h^{\gamma} .
\end{aligned}
$$

Taking the supremum, we find

$$
\sup _{i, j \in \mathbb{Z}}\left|u_{i, j}^{n+1}\right| \leq \sup _{i, j}\left|u_{i, j}^{n}\right|+C h^{\gamma}, \quad n \in \mathbb{N},
$$

and by induction

$$
\sup _{i, j \in \mathbb{Z}}\left|u_{i, j}^{n+1}\right| \leq \sup _{i, j \in \mathbb{Z}}\left|u_{i, j}^{0}\right|+C n h^{\gamma}, \quad n \in \mathbb{N} .
$$

Because of $n \cdot h \leq T$ and (4.9c), the result follows.

Theorem 4.1 yields the $L^{\infty}$-stability of the scheme (4.3)-(4.5) for the usual choices of antidiffusive fluxes. For the sake of definiteness, we tacitly assume from now on that the scheme under consideration admits an incremental decomposition of the form indicated in Theorem 4.1 in such a way that the scheme is stable in $L^{\infty}$-norm. In particular, in the case of the scheme with the flux limiter (4.8), a direct application of Theorem 4.1 leads to the following result whose proof is immediate. Actually, the same result of stability can be obtained for the general flux limiter schemes. We omit the proof.

Corollary 4.1 ( $L^{\infty}$-estimate for a scheme with flux limiter). The uniformly accurate version of the Van Leer flux limiter (see (4.8)) for an equation with several space variables is stable in $L^{\infty}$-norm, provided that $\lambda_{x}$ and $\lambda_{y}$ satisfy the CFL condition

$$
\lambda_{x} \sup _{u}\left|\mathbf{f}^{\prime}(u)\right| \leq \frac{1}{4}, \quad \lambda_{y} \sup _{u}\left|\mathbf{g}^{\prime}(u)\right| \leq \frac{1}{4},
$$

the supremum being taken over all $u$ under consideration.

4.4. Convergence for an equation in one space dimension. In this subsection, we consider the case of an equation with only one space variable. In fact, when the initial data of problem (1.1), (1.2) does not depend on the variable $y$, then both the exact solution $u$ to problem (1.1), (1.2) and the approximate solution $u^{h}$ defined by (2.9) and (4.3)-(4.5) do not depend on $y$. As a consequence, we can use exactly the same notation as above just omitting the index $j$. In particular, (4.3) and (4.4) become

$$
u_{i}^{n+1}=u_{i}^{n}-\lambda_{x}\left(f_{i+1 / 2}^{n}-f_{i-1 / 2}^{n}\right), \quad n \in \mathbb{N}, i \in \mathbb{Z},
$$


and

$$
f_{i+1 / 2}^{n}=p_{i+1 / 2}^{n}+\frac{1}{\lambda_{x}} a_{i+1 / 2}^{n}, \quad n \in \mathbb{N}, i \in \mathbb{Z} .
$$

The approximate solutions will be denoted interchangeably by $\left\{u^{h}\right\}$ or $\left(u_{i}^{n}\right)$. To apply the general method of $\S 2$, we need to obtain the weak estimate of the discrete space derivatives of the approximate solutions. For this purpose, following the ideas developed in $\S 3$, we begin with an evaluation of the amount of entropy production generated by the scheme. The following lemma is a generalization of Theorem 3.3 to the high-order accurate schemes under consideration. Recall again that for simplicity the initial data $u_{0}$ is assumed to be a measurable and bounded function with compact support.

Lemma 4.1 (A sharp approximate entropy inequality). Suppose that the CFL condition

$$
\lambda_{x} \sup _{u}\left|\mathbf{f}^{\prime}(u)\right| \leq \frac{1}{2}
$$

holds true (the supremum is taken over all $u$ under consideration) and the antidiffusive flux in (4.13) satisfies the condition

$$
\left|a_{i+1 / 2}^{n}\right| \leq M h^{\alpha}, \quad n \in \mathbb{N}, i \in \mathbb{Z}, \text { with } M>0 \text { and } \alpha \in\left(\frac{2}{3}, 1\right) .
$$

Then the scheme (4.12), (4.13) satisfies the following sharp entropy inequality $(n \in \mathbb{N}$ with $n h \leq T)$ :

$$
\sum_{i \in \mathbb{Z}} \frac{1}{2}\left(u_{i}^{n+1}\right)^{2} h_{x}-\sum_{i \in \mathbb{Z}} \frac{1}{2}\left(u_{i}^{n}\right)^{2} h_{x}+K_{1} \sum_{i \in \mathbb{Z}} \lambda_{x}\left|u_{i+1}^{n}-u_{i}^{n}\right|^{3} h_{x} \leq K_{2} h^{3 \alpha / 2},
$$

where the constants $K_{1}$ and $K_{2}$ are positive and independent of $h, \lambda_{x}$, and $u_{0}$ (but can depend on $T$ and $\mathbf{f}$ ).

Remark 4.1. We notice that in fact no property of $L^{\infty}$ uniform stability is used to derive inequality (4.16).

Proof of Lemma 4.1. We introduce the notation

$$
\bar{u}_{i}^{n+1}=u_{i}^{n}-\lambda_{x}\left(p_{i+1 / 2}^{n}-p_{i-1 / 2}^{n}\right), \quad n \in \mathbb{N}, \quad i \in \mathbb{Z},
$$

and observe that (4.12) can be rewritten in the form

$$
u_{i}^{n+1}=\bar{u}_{i}^{n+1}-\left(a_{i+1 / 2}^{n}-a_{i-1 / 2}^{n}\right), \quad n \in \mathbb{N}, i \in \mathbb{Z} .
$$

We thus get

$$
\begin{aligned}
\frac{1}{2}\left(u_{i}^{n+1}\right)^{2}= & \frac{1}{2}\left(\bar{u}_{i}^{n+1}\right)^{2}+\frac{1}{2}\left(a_{i+1 / 2}^{n}-a_{i-1 / 2}^{n}\right)^{2} \\
& -\bar{u}_{i}^{n+1}\left(a_{i+1 / 2}^{n}-a_{i-1 / 2}^{n}\right), \quad n \in \mathbb{N}, \quad i \in \mathbb{Z},
\end{aligned}
$$

which leads us to set

$$
R^{n}=\sum_{i \in \mathbb{Z}} \frac{1}{2}\left(a_{i+1 / 2}^{n}-a_{i-1 / 2}^{n}\right)^{2} h_{x}-\sum_{i \in \mathbb{Z}} \bar{u}_{i}^{n+1}\left(a_{i+1 / 2}^{n}-a_{i-1 / 2}^{n}\right) h_{x}, \quad n \in \mathbb{N},
$$


so that we have

$$
\begin{aligned}
\sum_{i \in \mathbb{Z}} & \frac{1}{2}\left(u_{i}^{n+1}\right)^{2} h_{x}-\sum_{i \in \mathbb{Z}} \frac{1}{2}\left(u_{i}^{n}\right)^{2} h_{x} \\
= & \sum_{i \in \mathbb{Z}} \frac{1}{2}\left(\bar{u}_{i}^{n+1}\right)^{2} h_{x}-\sum_{i \in \mathbb{Z}} \frac{1}{2}\left(u_{i}^{n}\right)^{2} h_{x}+R^{n}, \quad n \in \mathbb{N} .
\end{aligned}
$$

By using Theorem 3.3 of $\S 3$, equality (4.21) implies

$$
\begin{aligned}
& \sum_{i \in \mathbb{Z}} \frac{1}{2}\left(u_{i}^{n+1}\right)^{2} h_{x}-\sum_{i \in \mathbb{Z}} \frac{1}{2}\left(u_{i}^{n}\right)^{2} h_{x} \\
& \quad \leq-K \sum_{i \in \mathbb{Z}} \lambda_{x}\left|u_{i+1}^{n}-u_{i}^{n}\right|^{3} h_{x}+R^{n}, \quad n \in \mathbb{N} .
\end{aligned}
$$

In order to estimate the term $R^{n}$, we recall that under the CFL restriction (4.14) the $E$-scheme (4.18) satisfies the TVD property

$$
\sum_{i \in \mathbb{Z}}\left|\bar{u}_{i+1}^{n+1}-\bar{u}_{i}^{n+1}\right| \leq \sum_{i \in \mathbb{Z}}\left|u_{i+1}^{n}-u_{i}^{n}\right|, \quad n \in \mathbb{N} .
$$

From (4.20) we then deduce

$$
\begin{aligned}
\left|R^{n}\right| & \leq \sum_{i \in \mathbb{Z}} 2 \max \left(\left|a_{i+1 / 2}^{n}\right|^{2},\left|a_{i-1 / 2}^{n}\right|^{2}\right) h_{x}+\sum_{i \in \mathbb{Z}}\left|a_{i-1 / 2}^{n}\right|\left|\bar{u}_{i+1}^{n+1}-\bar{u}_{i}^{n+1}\right| h_{x} \\
& \leq \sum_{i \in \mathbb{Z}} 2 \max \left(\left|a_{i+1 / 2}^{n}\right|^{2},\left|a_{i-1 / 2}^{n}\right|^{2}\right) h_{x}+\max _{i \in \mathbb{Z}}\left|a_{i-1 / 2}^{n}\right| \sum_{i \in \mathbb{Z}}\left|u_{i+1}^{n}-u_{i}^{n}\right| h_{x} .
\end{aligned}
$$

But using now the condition (4.15), and since the initial data has compact support and the numerical flux is assumed to be consistent, we necessarily have $a_{i+1 / 2}^{n}=0$ for $i$ large enough. We thus find the estimate

$$
\left|R^{n}\right| \leq C h^{2 \alpha}+M h^{\alpha} \sum_{i \in \mathbb{Z}}\left|u_{i+1}^{n}-u_{i}^{n}\right| h_{x}, \quad n \in \mathbb{N} .
$$

Then (4.22) and (4.24) yield the following inequality $(K>0$ is given by Theorem 3.4 and $M>0$ by (4.15)):

$$
\begin{aligned}
& \sum_{i \in \mathbb{Z}} \frac{1}{2}\left(u_{i}^{n+1}\right)^{2} h_{x}-\sum_{i \in \mathbb{Z}} \frac{1}{2}\left(u_{i}^{n}\right)^{2} h_{x} \\
& \leq-K \sum_{i \in \mathbb{Z}} \lambda_{x}\left|u_{i+1}^{n}-u_{i}^{n}\right|^{3} h_{x}+M h^{\alpha} \sum_{i \in \mathbb{Z}}\left|u_{i+1}^{n}-u_{i}^{n}\right| h_{x}+C h^{2 \alpha}
\end{aligned}
$$

We finally remark that

$$
\sum_{i \in \mathbb{Z}} M h^{\alpha}\left|u_{i+1}^{n}-u_{i}^{n}\right| h_{x} \leq \frac{2}{3}\left(\frac{M h^{\alpha}}{K^{1 / 3}}\right)^{3 / 2}+\frac{K}{3} \sum_{i \in \mathbb{Z}}\left|u_{i+1}^{n}-u_{i}^{n}\right|^{3} h_{x},
$$

so that $(4.25)$ gives

$$
\begin{aligned}
\sum_{i \in \mathbb{Z}} & \frac{1}{2}\left(u_{i}^{n+1}\right)^{2} h_{x}-\sum_{i \in \mathbb{Z}} \frac{1}{2}\left(u_{i}^{n}\right)^{2} h_{x} \\
\leq & -\frac{2 K}{3} \sum_{i \in \mathbb{Z}} \lambda_{x}\left|u_{i+1}^{n}-u_{i}^{n}\right|^{3} h_{x}+C h^{3 \alpha / 2} .
\end{aligned}
$$

This completes the proof of the lemma. 
From Lemma 4.1, we deduce:

Lemma 4.2 (Uniform estimate of the discrete space derivative). Under the CFL stability condition (4.14) and the condition (4.15) on the antidiffusive flux, the scheme (4.12), (4.13) satisfies the uniform estimate $\left(\lambda_{x}=h / h_{x}\right.$ kept constant)

$$
h^{2 / 3} \sum_{\substack{n \in \mathbb{N} \\ n h \leq T}} \sum_{i \in \mathbb{Z}}\left|\frac{u_{i+1}^{n}-u_{i}^{n}}{h_{x}}\right| h h_{x} \leq C,
$$

where $C$ is a constant independent of $h$ (but dependent in general on $\lambda_{x}, u_{0}, T$, and $\mathbf{f}$ ).

Proof. By summation of (4.16), we have

$$
\begin{aligned}
\sum_{i \in \mathbb{Z}} \frac{1}{2}\left(u_{i}^{n+1}\right)^{2} h_{x}-\sum_{i \in \mathbb{Z}} \frac{1}{2}\left(u_{i}^{0}\right)^{2} h_{x} \\
+C \sum_{m=0}^{n} \sum_{i \in \mathbb{Z}} \lambda_{x}\left|u_{i+1}^{m}-u_{i}^{m}\right|^{3} h_{x} \leq C h^{3 \alpha / 2-1}
\end{aligned}
$$

for every $n \in \mathbb{N}$ with $n \cdot h \leq T$; thus,

$$
\sum_{\substack{n \in \mathbb{N} \\ n h \leq T}} \sum_{i \in \mathbb{Z}}\left|u_{i+1}^{n}-u_{i}^{n}\right|^{3} h \leq C .
$$

If $[r, s]$ denotes a set containing the support of the initial data $\left(u_{i}^{0}\right)_{i \in \mathbb{Z}}$, then, for each $n$, the support of the sequence $\left(u_{i}^{n}\right)_{i \in \mathbb{N}}$ is included in the set $\left[r-n h_{x}, s+n h_{x}\right]$. Thus, using this remark and the Hölder inequality, we deduce from (4.29) that

$$
\begin{aligned}
\sum_{\substack{n \in \mathbb{N} \\
n h \leq T}} \sum_{i \in \mathbb{Z}}\left|u_{i+1}^{n}-u_{i}^{n}\right| h_{x} h & \leq\left(L_{x} T\right)^{2 / 3}\left(\sum_{\substack{n \in \mathbb{N} \\
n h \leq T}} \sum_{i \in \mathbb{Z}}\left|u_{i+1}^{n}-u_{i}^{n}\right|^{3} h_{x} h\right)^{1 / 3} \\
& \leq C h_{x}^{1 / 3},
\end{aligned}
$$

where $L_{x}$ denotes a bound independent of $h$ of the length of the support of $u^{h}$, hence

$$
h_{x}^{2 / 3} \sum_{\substack{n \in \mathbb{N} \\ n h \leq T}} \sum_{i \in \mathbb{Z}}\left|u_{i+1}^{n}-u_{i}^{n}\right| h \leq C .
$$

Since $\lambda_{x}$ is kept constant, (4.27) is a consequence of (4.30).

Using the uniform estimate given by Lemma 4.2, we now derive discrete entropy inequalities satisfied by the scheme (for the notation below, see $\S 2$, Theorem 2.3). The idea of the proof below is to get these entropy inequalities by perturbation of the entropy inequality satisfied by the underlying $E$-scheme. 
Condition (4.15) turns out to be essential here. This idea was already used in another context by Leroux [30], Leroux and Quesseveur [32], and Vila [50]; but let us recall that these authors needed a BV-estimate, which is not necessary in the present approach.

Lemma 4.3 (Approximate entropy inequalities). Suppose that the conditions (4.14) and (4.15) are satisfied. Let $U: \mathbb{R} \rightarrow \mathbb{R}$ be a convex function of class $C^{2}$, and $F$ be a numerical entropy flux associated with the given numerical E-flux $p: \mathbb{R}^{2 k} \rightarrow \mathbb{R}$. Assume moreover that the family of approximate solutions remains uniformly bounded in $L^{\infty}$-norm (cf. Theorem 4.1). Then the scheme satisfies an inequality of the form

$$
\frac{1}{h}\left(U_{i}^{n+1}-U_{i}^{n}\right)+\frac{1}{h_{x}}\left(F_{i+1 / 2}^{n}-F_{i-1 / 2}^{n}\right) \leq R_{i}^{n}, \quad n \in \mathbb{N}, i \in \mathbb{Z},
$$

where the term $R_{i}^{n}$ tends to zero in the following sense:

$$
\sum_{\substack{n \in \mathbb{N} \\ n h \leq T}} \sum_{i \in \mathbb{Z}} \varphi\left(t_{n}, x_{i}\right) R_{i}^{n} h_{x} h \rightarrow 0, \quad \text { when } h \rightarrow 0,
$$

for every $C^{1}$ function $\varphi:(0, T) \times \mathbb{R} \rightarrow \mathbb{R}_{+}$with compact support.

Proof. It is well known that an $E$-scheme satisfies an exact entropy inequality in each cell (Osher [35]). So, using the notation introduced in the proof of Lemma 4.1 (see (4.17) and (4.18)), we have

$$
U\left(\bar{u}_{i}^{n+1}\right)-U\left(u_{i}^{n}\right)+\lambda_{x}\left(F_{i+1 / 2}^{n}-F_{i-1 / 2}^{n}\right) \leq 0, \quad n \in \mathbb{N}, i \in \mathbb{Z} .
$$

We thus obtain

$U\left(u_{i}^{n+1}\right)-U\left(u_{i}^{n}\right)+\lambda_{x}\left(F_{i+1 / 2}^{n}-F_{i-1 / 2}^{n}\right) \leq U\left(u_{i}^{n+1}\right)-U\left(\bar{u}_{i}^{n+1}\right), \quad n \in \mathbb{N}, i \in \mathbb{Z}$,

i.e., dividing by $h$,

$$
\frac{1}{h}\left(U_{i}^{n+1}-U_{i}^{n}\right)+\frac{1}{h_{x}}\left(F_{i+1 / 2}^{n}-F_{i-1 / 2}^{n}\right) \leq R_{i}^{n}, \quad n \in \mathbb{R}, i \in \mathbb{Z},
$$

which is exactly (4.31) if we set

$$
R_{i}^{n}=\frac{1}{h}\left(U\left(u_{i}^{n+1}\right)-U\left(\bar{u}_{i}^{n+1}\right)\right), \quad n \in \mathbb{N}, i \in \mathbb{Z} .
$$

Formula (4.18) and the convexity of the function $U$ give

$$
R_{i}^{n} \leq \frac{1}{h} U^{\prime}\left(u_{i}^{n+1}\right)\left(u_{i}^{n+1}-\bar{u}_{i}^{n+1}\right)=-\frac{1}{h} U^{\prime}\left(u_{i}^{n+1}\right)\left(a_{i+1 / 2}^{n}-a_{i-1 / 2}^{n}\right) .
$$

Introducing a positive test function $\varphi$ and setting $\varphi_{i}^{n}=\varphi\left(t_{n}, x_{i}\right)$, we deduce from the above inequality that

$$
\sum_{\substack{n \in \mathbb{N} \\ n h \leq T}} \sum_{i \in \mathbb{Z}} \varphi_{i}^{n} R_{i}^{n} h_{x} h \leq-\frac{1}{\lambda_{x}} \sum_{\substack{n \in \mathbb{N} \\ n h \leq T}} \sum_{i \in \mathbb{Z}} \varphi_{i}^{n} U^{\prime}\left(u_{i}^{n+1}\right)\left(a_{i+1 / 2}^{n}-a_{i-1 / 2}^{n}\right) h .
$$


We thus get

$$
\sum_{\substack{n \in \mathbb{N} \\ n h \leq T}} \sum_{i \in \mathbb{Z}} \varphi_{i}^{n} R_{i}^{n} h_{x} h \leq C_{1}+C_{2}
$$

where we have set

$$
C_{1}(h)=\frac{1}{\lambda_{x}} \sum_{\substack{n \in \mathbb{N} \\ n h \leq T}} \sum_{i \in \mathbb{Z}} a_{i+1 / 2}^{n} U^{\prime}\left(u_{i+1}^{n+1}\right)\left(\varphi_{i+1}^{n}-\varphi_{i}^{n}\right) h
$$

and

$$
C_{2}(h)=\frac{1}{\lambda_{x}} \sum_{\substack{n \in \mathbb{N} \\ n h \leq T}} \sum_{i \in \mathbb{Z}} a_{i+1 / 2}^{n} \varphi_{i}^{n}\left(U^{\prime}\left(u_{i+1}^{n+1}\right)-U^{\prime}\left(u_{i}^{n+1}\right)\right) h .
$$

To estimate the term $C_{1}(h)$, we use the condition (4.15) on the antidiffusive flux and the $L^{\infty}$-estimate and obtain

$$
C_{1}(h) \leq C h^{\alpha} \sum_{\substack{n \in \mathbb{N} \\ n h \leq T}} \sum_{i \in \mathbb{Z}} \frac{\left|\varphi_{i+1}^{n}-\varphi_{i}^{n}\right|}{h_{x}} h_{x} h,
$$

so

$$
C_{1}(h) \leq C\left\|\varphi_{x}\right\|_{L^{\infty}} h^{\alpha} \rightarrow 0 .
$$

On the other hand, estimate (4.27) of Lemma 4.2 allows us to bound $C_{2}(h)$ as follows:

$$
C_{2}(h) \leq C h^{\alpha}\|\varphi\|_{L^{\infty}}\left\|U^{\prime \prime}\left(u_{h}\right)\right\|_{L^{\infty}} \sum_{\substack{n \in \mathbb{N} \\ n h \leq T}} \sum_{i \in \mathbb{Z}}\left|u_{i+1}^{n+1}-u_{i}^{n+1}\right| h
$$

thus,

$$
C_{2}(h) \leq C\|\varphi\|_{L^{\infty}} h^{\alpha-2 / 3} \rightarrow 0,
$$

since $\alpha>\frac{2}{3}$ by (4.15). Finally, (4.32) follows from (4.33)-(4.35).

We can now apply the method of proof of $\S 2$. In view of the uniform $L^{\infty}$ estimate (4.10) obtained in Theorem 4.1, we construct from the family $\left\{u^{h}\right\}$ a Young measure denoted by $\nu:(0, T) \times \mathbb{R} \rightarrow \operatorname{Prob}(\mathbb{R})$ which represents all the weak limits of the composite functions of $u^{h}$. Since Lemma 4.2 yields a uniform estimate of the space derivatives $((4.27)$ is of the form (2.15) with $\beta=\frac{2}{3}$ ), Theorem 2.2 applies and shows that $\nu$ is a measure-valued solution to the conservation law (1.1). Lemma 4.3 and Theorem 2.3 then prove that $\nu$ is an entropy measure-valued solution. Moroever, Theorem 2.4 applies easily since Lemma 4.3 can be used with a given strictly convex function (for instance $U(u)=u^{2} / 2$; but we could also do for this point with Lemma 4.1); thus, the Young measure $\nu$ satisfies the initial data $u_{0}$. Finally, Theorem 2.1 shows that $\nu$ reduces to a Dirac mass. The conclusion is summarized in the following theorem. 
Theorem 4.2 (Convergence of the difference schemes with antidiffusive flux). Consider the family of approximate solutions $u^{h}$ constructed by the scheme with antidiffusive flux (4.12), (4.13) (and (2.9), (2.10)) for a conservation law in one space dimension. Assume that the family $\left\{u^{h}\right\}$ is uniformly bounded in $L^{\infty}$. norm (cf. Theorem 4.1). Then under the CFL stability condition (4.14) and the condition (4.15) on the antidiffusive flux, the Young measure $\nu$ associated with the family $\left\{u^{h}\right\}$ reduces to a Dirac mass, i.e.,

$$
\nu_{t, x}=\delta_{u(t, x)}, \text { a.e. } t \in(0, T), x \in \mathbb{R},
$$

holds true, where $u$ is the unique entropy weak $L^{\infty}$-solution to problem (1.1), (1.2) (in one space dimension). Hence, when $h$ goes to zero, the sequence $\left\{u^{h}\right\}_{h \geq 0}$ converges in the $L^{1}$ strong topology to the unique entropy solution $u$.

This result is essentially the same as the one obtained by the TVB approach. But we emphasize that the proofs of this result are new and their interest lies in the fact that they can be generalized to equations with several space variables. Moreover, we point out that this result gives in particular the convergence of the scheme with flux limiter (4.8) introduced in $\S 4.2$ under the condition $\alpha_{1}, \beta_{1} \in$ $\left(\frac{2}{3}, 1\right)$ and $\alpha_{2}, \beta_{2} \in[1,2]$ (instead of $\alpha_{1}, \beta_{1} \in(0,1)$ and $\alpha_{2}, \beta_{2}=2$ by the TVB approach). See Corollary 4.2 below for a precise statement of convergence.

4.5. Convergence for an equation with several space variables. The previous analysis of convergence is now generalized to the case of an equation with several space variables. The steps of the proof here are exactly the same as the ones of $\S 4.4$ and are based on the general theorems of convergence recalled in $\S 2$ and the sharp entropy inequality derived in $\S 3$.

The notation was introduced in (4.3)-(4.6) (see also (2.9), (2.10)). As in Lemmas 4.1 and 4.2, the estimate of the discrete space derivatives of $u^{h}$ is derived via an evaluation of the total amount of entropy production of the scheme.

Lemma 4.4 (Uniform estimate of the discrete space derivative). Assume that the CFL stability condition

$$
\lambda_{x} \sup _{u}\left|\mathbf{f}^{\prime}(u)\right| \leq \frac{1}{4}, \quad \lambda_{y} \sup _{u}\left|\mathbf{g}^{\prime}(u)\right| \leq \frac{1}{4}
$$

(the supremum being taken over all $u$ under consideration) and the following condition on the antidiffusive fluxes,

$$
\begin{array}{ll}
\left|a_{i+1 / 2, j}^{n}\right| \leq C h^{\alpha}, & n \in \mathbb{N}, i, j \in \mathbb{Z}, \text { with } \alpha \in\left(\frac{2}{3}, 1\right), \\
\left|b_{i, j+1 / 2}^{n}\right| \leq C h^{\beta}, & n \in \mathbb{N}, i, j \in \mathbb{Z}, \text { with } \beta \in\left(\frac{2}{3}, 1\right),
\end{array}
$$

are satisfied. Then the scheme (4.3)-(4.5) satisfies the following entropy 
inequality:

$$
\begin{aligned}
& \sum_{i, j \in \mathbb{Z}} \frac{1}{2}\left(u_{i, j}^{n+1}\right)^{2} h_{x} h_{y}-\sum_{i, j \in \mathbb{Z}} \frac{1}{2}\left(u_{i, j}^{n}\right)^{2} h_{x} h_{y} \\
& \quad+K_{1} \sum_{i, j \in \mathbb{Z}} \lambda_{x}\left|u_{i+1, j}^{n}-u_{i, j}^{n}\right|^{3} h_{x} h_{y} \\
& \quad+K_{2} \sum_{i, j \in \mathbb{Z}} \lambda_{y}\left|u_{i, j+1}^{n}-u_{i, j}^{n}\right|^{3} h_{x} h_{y} \leq C h^{3 \gamma / 2}, \quad n \in \mathbb{N},
\end{aligned}
$$

where $\gamma=\min (\alpha, \beta)$ and $K_{1}, K_{2}$ are two positive constants independent of $h, \lambda_{x}$ and $u_{0}$.

Proof. The proof is based on the following (convex) decomposition of the scheme into two one-dimensional schemes:

$$
\begin{aligned}
u_{i, j}^{n+1}= & \frac{1}{2}\left\{u_{i, j}^{n}-2 \lambda_{x}\left(f_{i+1 / 2, j}^{n}-f_{i-1 / 2, j}^{n}\right)\right\} \\
& +\frac{1}{2}\left\{u_{i, j}^{n}-2 \lambda_{y}\left(g_{i, j+1 / 2}^{n}-g_{i, j-1 / 2}^{n}\right)\right\}, \quad n \in \mathbb{N}, i, j \in \mathbb{Z} .
\end{aligned}
$$

Since the function $\frac{1}{2} u^{2}$ is convex, we have

$$
\begin{aligned}
\frac{1}{2}\left(u_{i, j}^{n+1}\right)^{2} \leq & \frac{1}{4}\left\{u_{i, j}^{n}-2 \lambda_{x}\left(f_{i+1 / 2, j}^{n}-f_{i-1 / 2, j}^{n}\right)\right\}^{2} \\
& +\frac{1}{4}\left\{u_{i, j}^{n}-2 \lambda_{y}\left(g_{i, j+1 / 2}^{n}-g_{i, j-1 / 2}^{n}\right)\right\}^{2}, \quad n \in \mathbb{N}, i, j \in \mathbb{Z} .
\end{aligned}
$$

We thus get

$$
\frac{1}{2}\left(u_{i, j}^{n+1}\right)^{2}-\frac{1}{2}\left(u_{i, j}^{n}\right)^{2} \leq A_{i, j}^{n}+B_{i, j}^{n}, \quad n \in \mathbb{N}, i, j \in \mathbb{Z},
$$

where we have set

$$
A_{i, j}^{n}=\frac{1}{2}\left(\frac{1}{2}\left\{u_{i, j}^{n}-2 \lambda_{x}\left(f_{i+1 / 2, j}^{n}-f_{i-1 / 2, j}^{n}\right)\right\}^{2}-\frac{1}{2}\left(u_{i, j}^{n}\right)^{2}\right), \quad n \in \mathbb{N}, i, j \in \mathbb{Z},
$$

and

$$
B_{i, j}^{n}=\frac{1}{2}\left(\frac{1}{2}\left\{u_{i, j}^{n}-2 \lambda_{y}\left(g_{i, j+1 / 2}^{n}-g_{i, j-1 / 2}^{n}\right)\right\}^{2}-\frac{1}{2}\left(u_{i, j}^{n}\right)^{2}\right), \quad n \in \mathbb{N}, i, j \in \mathbb{Z} .
$$

Taking into account (4.37) and (4.38), a direct application of Lemma 4.1 leads to the inequality

$$
\begin{aligned}
\sum_{i \in \mathbb{Z}} & \frac{1}{2}\left\{u_{i, j}^{n}-2 \lambda_{x}\left(f_{i+1 / 2, j}^{n}-f_{i-1 / 2, j}^{n}\right)\right\}^{2} h_{x}-\sum_{i \in \mathbb{Z}} \frac{1}{2}\left(u_{i, j}^{n}\right)^{2} h_{x} \\
& +C \sum_{i \in \mathbb{Z}} 2 \lambda_{x}\left|u_{i+1, j}^{n}-u_{i, j}^{n}\right|^{3} h_{x} \leq C h^{3 \alpha / 2}
\end{aligned}
$$

for each time $n \quad(n \in \mathbb{N}, n \cdot h \leq T)$ and each $y$-index $j \in \mathbb{Z}$. But the support of $\left(u_{i, j}^{n}\right)$ is included in a set of the form $\{(i, j) /|i|,|j| \leq N+n\}$ (if the support of $\left(u_{i, j}^{0}\right)$ is included in the set $\left.\{(i, j) /|i|,|j| \leq N\}\right)$. Moreover, $A_{i, j}^{n}$ is zero for $(i, j)$ outside this set, so that $(4.41)$ implies, after multiplication by $h_{y}$ and summation with respect to $j$,

$$
\frac{1}{2} \sum_{i, j \in \mathbb{Z}} A_{i, j}^{n} h_{x} h_{y}+C 2 \lambda_{x} \sum_{i, j \in \mathbb{Z}}\left|u_{i+1, j}^{n}-u_{i, j}^{n}\right|^{3} h_{x} h_{y} \leq C h^{3 / 2 \alpha}, \quad n \in \mathbb{N} .
$$


We thus have

$$
\sum_{i, j \in \mathbb{Z}} A_{i, j}^{n} h_{x} h_{y}+C \sum_{i, j \in \mathbb{Z}} \lambda_{x}\left|u_{i+1, j}^{n}-u_{i, j}^{n}\right|^{3} h_{x} h_{y} \leq C h^{3 \alpha / 2},
$$

$$
n \in \mathbb{N}, n h \leq T
$$

Similarly, for the $B_{i, j}^{n}$ terms we obtain

$$
\begin{aligned}
& \sum_{i, j \in \mathbb{Z}} B_{i, j}^{n} h_{x} h_{y}+C \sum_{i, j \in \mathbb{Z}} \lambda_{y}\left|u_{i, j+1}^{n}-u_{i, j}^{n}\right|^{3} h_{x} h_{y} \leq C h^{3 \beta / 2}, \\
& n \in \mathbb{N}, \quad n h \leq T .
\end{aligned}
$$

The inequality (4.39) follows immediately from (4.40), (4.42), and (4.43).

From Lemma 4.4 we deduce the estimate of the space derivatives. The proof is similar to the one of Lemma 4.2, so we omit it.

Lemma 4.5 (Uniform estimate of the discrete space derivatives). Under the assumptions of Lemma 4.4, we have the uniform estimate

$$
h^{2 / 3} \sum_{\substack{n \in \mathbb{N} \\ n h \leq T}} \sum_{i, j \in \mathbb{Z}}\left(\frac{\left|u_{i+1, j}^{n}-u_{i, j}^{n}\right|}{h_{x}}+\frac{\left|u_{i, j+1}^{n}-u_{i, j}^{n}\right|}{h_{y}}\right) h_{x} h_{y} h \leq C .
$$

Finally, we derive approximate entropy inequalities satisfied by the scheme.

Lemma 4.6 (Approximate entropy inequalities). Suppose that the CFL stability condition (4.37) and the condition (4.38) are satisfied. Let $U: \mathbb{R} \rightarrow \mathbb{R}$ be a convex function of class $C^{2}$ and let $(F, G)$ denote the numerical entropy flux functions associated with $p$ and $q$. Assume moreover that $\left\{u^{h}\right\}$ remains uniformly bounded in $L^{\infty}$-norm. Then the scheme satisfies the entropy inequality of the form (2.16), (2.17) (see Theorem 2.3).

Proof of Lemma 4.6. Let us introduce the notation

$$
\begin{aligned}
\bar{u}_{i j}^{n+1}= & u_{i j}^{n}-\lambda_{x}\left(p_{i+1 / 2, j}^{n}-p_{i-1 / 2, j}^{n}\right) \\
& -\lambda_{y}\left(q_{i, j+1 / 2}^{n}-q_{i, j-1 / 2}^{n}\right), \quad n \in \mathbb{N}, i, j \in \mathbb{Z},
\end{aligned}
$$

so that with (4.3)-(4.5), we obtain

$$
\begin{array}{r}
u_{i j}^{n+1}=\bar{u}_{i j}^{n+1}-\left(a_{i+1 / 2, j}^{n}-a_{i-1 / 2, j}^{n}\right)-\left(b_{i, j+1 / 2}^{n}-b_{i, j-1 / 2}^{n}\right), \\
n \in \mathbb{N}, i, j \in \mathbb{Z} .
\end{array}
$$

Since an $E$-scheme in several space dimensions satisfies an exact cell entropy inequality (Osher [35]), (4.45) gives

$$
\begin{array}{r}
U\left(\bar{u}_{i j}^{n+1}\right)-U\left(u_{i j}^{n}\right)+\lambda_{x}\left(F_{i+1 / 2, j}^{n}-F_{i-1 / 2, j}^{n}\right)+\lambda_{y}\left(G_{i, j+1 / 2}^{n}-G_{i, j-1 / 2}^{n}\right) \leq 0, \\
n \in \mathbb{N}, i, j \in \mathbb{Z} .
\end{array}
$$


Thus, subtracting $U\left(u_{i j}^{n+1}\right)=U_{i j}^{n+1}$ and dividing by $h$, we get

$$
\begin{aligned}
& \frac{1}{h}\left(U_{i, j}^{n+1}-U_{i, j}^{n}\right)+\frac{1}{h_{x}}\left(F_{i+1 / 2, j}^{n}-F_{i-1 / 2, j}^{n}\right) \\
& \quad+\frac{1}{h_{y}}\left(G_{i, j+1 / 2}^{n}-G_{i, j-1 / 2}^{n}\right) \leq R_{i j}^{n}, \quad n \in \mathbb{N}, i, j \in \mathbb{Z},
\end{aligned}
$$

where we have set

$$
R_{i j}^{n}=\frac{1}{h}\left(U\left(u_{i}^{n+1}\right)-U\left(\bar{u}_{i}^{n+1}\right)\right), \quad n \in \mathbb{N}, i, j \in \mathbb{Z} .
$$

Inequality (4.47) is exactly (2.16). It remains to prove that the $R_{i j}^{n}$ terms satisfy (2.17). Since the function $U$ is convex, it results from (4.46) that

$$
\begin{aligned}
& R_{i j}^{n} \leq-\frac{1}{h} U^{\prime}\left(u_{i j}^{n+1}\right)\left(\left(a_{i+1 / 2, j}^{n}-a_{i-1 / 2, j}^{n}\right)+\left(b_{i, j+1 / 2}^{n}-b_{i, j-1 / 2}^{n}\right)\right), \\
& n \in \mathbb{N}, i, j \in \mathbb{Z} .
\end{aligned}
$$

Then, introducing a positive test function $\varphi$ and using the estimate (4.44) together with the condition (4.38) on the antidiffusive fluxes, the same arguments as in the proof of Lemma 4.3 show the inequality

$$
\sum_{\substack{n \in \mathbb{N} \\ n h \leq T}} \sum_{i, j \in \mathbb{Z}} \varphi\left(t_{n}, x_{i}, y_{j}\right) R_{i j}^{n} h_{x} h_{y} h \leq C h^{\gamma-2 / 3}
$$

with $\gamma=\min (\alpha, \beta)$. That completes the proof.

Finally, as in $\S 4.4$, using the general convergence theorems (Theorems 2.22.4) of $\S 2$ and the uniqueness theorem of Di Perna (Theorem 2.1), we deduce from Lemmas 4.4-4.6 the following theorem, which is the main result of this paper.

Theorem 4.3 (Convergence of the difference schemes with antidiffusive fluxes). Consider the family of approximate solutions $\left\{u^{h}\right\}_{h>0}$ constructed by a scheme with antidiffusive fluxes, (4.3)-(4.5) and (2.9), (2.10), for a scalar conservation law in several space dimensions. Assume that this family is uniformly bounded in $L^{\infty}$-norm (cf. Theorem 4.1). Then under the CFL stability condition (4.37) and the condition (4.38) on the antidiffusive fluxes, the Young measure $\nu$ associated with the family $\left\{u^{h}\right\}$ reduces to a Dirac mass, i.e.,

$$
\nu_{t, x, y}=\delta_{u(t, x, y)} \text {, a.e. } t>0,(x, y) \in \mathbb{R}^{2},
$$

where $u$ denotes the unique entropy weak $L^{\infty}$ solution to problem (1.1), (1.2). Hence, when $h$ goes to zero, the approximate solutions $u^{h}$ converge to the unique entropy weak solution $u$ in the $L^{1}$ strong topology.

We recall that the $L^{\infty}$-estimate assumed in Theorem 4.3 is actually ensured by Theorem 4.1 for the main choices of antidiffusive fluxes introduced in the literature. In particular, in the case of the scheme constructed in $\S 4.2$ from the Van Leer flux limiter, we obtain the following convergence result (the proof is omitted). 
Corollary 4.2 (Convergence of a scheme with flux limiter). Under the CFL stability condition (4.11) and the condition

$$
\alpha_{1}, \beta_{1} \in\left(\frac{2}{3}, 1\right), \quad \alpha_{2}, \beta_{2} \in[1,2],
$$

the uniformly accurate version of the Van Leer scheme introduced in $\$ 4.2$ converges in the $L^{1}$-norm strongly towards the unique entropy weak $L^{\infty}$ solution of the problem (1.1), (1.2).

Remark 4.2. A number of generalizations of Theorem 4.3 are possible. For instance, if the $E$-schemes are chosen to be the modified Lax-Friedrichs scheme (see §3.3), then in view of Theorem 3.2 it is clear that Theorem 4.3 remains valid if (4.38) is replaced by the weaker condition

$$
\begin{array}{ll}
\left|a_{i+1 / 2, j}^{n}\right| \leq C h^{\alpha}, & n \in \mathbb{N}, i, j \in \mathbb{Z}, \text { with } \alpha \in\left(\frac{1}{2}, 1\right), \\
\left|b_{i, j+1 / 2}^{n}\right| \leq C h^{\beta}, & n \in \mathbb{N}, i, j \in \mathbb{Z}, \text { with } \beta \in\left(\frac{1}{2}, 1\right) .
\end{array}
$$

In particular, Corollary 4.2 remains true if instead of (4.50) we assume that

$$
\alpha_{1}, \beta_{1} \in\left(\frac{1}{2}, 1\right), \quad \alpha_{2}, \beta_{2} \in[1,2] .
$$

\section{BIBLIOGRAPHY}

1. J. Ball, $A$ version of the fundamental theorem for Young measures, Proc. Workshop on Continuum Theory of Phase Transitions (Nice, France, Jan. 1988) (M. Rascle and D. Serre, eds.), Lecture Notes in Phys., Springer, 1990.

2. J. P. Boris and D. L. Book, Flux-corrected transport. I: SHASTA, a fluid transport algorithm that works, J. Comput. Phys. 11 (1973), 38-69.

3. J. Cahouet and F. Coquel, Uniformly second order convergent schemes for hyperbolic conservation laws including Leonard's approach, Notes on Numerical Fluid Mechanics (J. Ballmann and R. Jeltsch, eds.), vol. 24, Vieweg, 1989.

4. B. Cockburn and C. W. Shu, The Runge-Kutta local projection $P^{1}$ discontinuous-Galerkin finite element method for scalar conservation laws, IMA Preprint Ser., no. 388, Univ. of Minnesota, 1988.

5. _ , TVB Runge-Kutta local projection discontinuous Galerkin finite element method for scalar conservation laws. II: General framework, Math. Comp. 52 (1989), 411-435.

6. $\mathrm{F}$. Coquel and $\mathrm{Ph}$. Le Floch, Convergence de schémas aux différences finies pour des lois de conservation à plusieurs variables d'espace, C. R. Acad. Sci. Paris (I) 310 (1990), 455-460.

7. __ Convergence of finite difference schemes for conservation laws in several space variables: general theory, SIAM Numer. Anal. (submitted).

8. M. Crandall and A. Majda, Monotone difference approximations for scalar conservation laws, Math. Comp. 34 (1980), 1-21.

9. B. Dacorogna, Weak continuity and weak lower semicontinuity of nonlinear functions, Lecture Notes in Math., vol. 922, Springer, Berlin, Heidelberg, New York, 1982.

10. S. F. Davis, TVD finite difference schemes and artificial viscosity, ICASE Report, No. 84-20, NASA, Langley Research Center, Hampton, VA, 1984.

11. __ A rotationally biased upwind difference scheme for the Euler equations, J. Comput. Phys. 56 (1984), 65-92.

12. Ding Xiaxi, Chen Giu-Qiang, Luo Peizhu, Convergence of the fractional step Lax-Friedrichs scheme and Godunov scheme for the isentropic system of gas dynamics, Comm. Math. Phys. 121 (1989), 63-84. 
13. R. J. Di Perna, Convergence of approximate solutions to conservation laws, Arch. Rational Mech. Anal. 82 (1983), 27-70.

14. _ Singularities and oscillations in solutions to conservation laws, Phys. D 12 (1984), 363-368.

15. __ Measure-valued solutions to conservation laws, Arch. Rational Mech. Anal. 88 (1985), 223-270.

16. Compensated compactness and general systems of conservation laws, Trans. Amer. Math. Soc. 292 (1985), 383-420.

17. E. Godlevski and P. A. Raviart, Introduction to the approximation of nonlinear hyperbolic problems, Graduate Course at University Paris VI, 1990.

18. S. K. Godunov, A difference method for numerical calculation of discontinuous solutions of the equations of hydrodynamics, Mat. Sb. 47 (89) (1959), 271-306. (Russian).

19. J. Goodman and P. D. Lax, On dispersive difference schemes. I, Comm. Pure Appl. Math. 41 (1988), 591-613.

20. J. B. Goodman and R. J. LeVeque, On the accuracy of stable schemes for $2 D$ scalar conservation laws, Math. Comp. 45 (1985), 15-21.

21. A. Harten, High resolution schemes for hyperbolic conservation laws, J. Comput. Phys. 49 (1983), 357-393.

22. S. N. Kružkov, First order quasilinear equations in several independent variables, Math. USSR-Sb. 10 (1970), 217-243.

23. O. A. Ladyženskaja and N. N. Ural'ceva, Boundary problems for linear and quasilinear parabolic equations, Amer. Math. Soc. Transl. Ser. 247 (1965), 217-299.

24. A. Lapidus, $A$ detached shock calculation by second-order finite differences, J. Comput. Phys. 2 (1967), 154-177.

25. P. D. Lax, Hyperbolic systems of conservation laws. II, Comm. Pure Appl. Math. 10 (1957), 537-566.

26. __ Shock waves and entropy, Contribution to Nonlinear Functional Analysis (E. A. Zarantonello, ed.), Academic Press, 1971, pp. 603-634.

27. __ Hyperbolic systems of conservation laws and the mathematical theory of shock waves, SIAM, Philadelphia, PA, 1973.

28. P. D. Lax and B. Wendroff, Systems of conservation laws, Comm. Pure Appl. Math. 13 (1960), 217-237.

29. __ Difference schemes for hyperbolic equations with high order of accuracy, Comm. Pure Appl. Math. 17 (1964), 381-398.

30. A. Y. Leroux, Approximation de quelques problemes hyperboliques nonlinéaires, Thesis, Univ. of Rennes, France, 1979.

31. _ Convergence of an accurate scheme for first order quasi linear equations, RAIRO, Numer. Anal. 15 (1981), 151-170.

32. A. Y. Leroux and P. Quesseveur, Convergence of an antidiffusive Lagrange-Euler scheme for quasilinear equations, SIAM J. Numer. Anal. 21 (1984), 985-994.

33. A. Majda and S. Osher, Numerical viscosity and entropy condition, Comm. Pure Appl. Math. 32 (1979), 797-838.

34. F. Murat, Compacité par compensation, Ann. Scuola Norm. Sup. Pisa Cl. Sci. (4) 5 (1978), 489-507.

35. S. Osher, Riemann solvers, the entropy condition and difference approximations, SIAM J. Numer. Anal. 21 (1984), 217-235.

36. S. Osher and S. Chakravarthy, High resolution schemes and the entropy condition, SIAM J. Numer. Anal. 21 (1984), 955-983.

37. R. Sanders, On convergence of monotone finite difference schemes with variable spatial differencing, Math. Comp. 40 (1983), 91-106. 
38. S. Schochet, Examples of measure-valued solutions, Comm. Partial Differential Equations 14 (1989), 545-576.

39. C. W. Shu, TVB uniformly high-order schemes for conservation laws, Math. Comp. 49 (1987), 105-121.

40. C. W. Shu and S. Osher, Efficient implementation of essentially non-oscillatory shock capturing schemes, J. Comput. Phys. 83 (1989), 32-78.

41. J. S. Smoller, Shock waves and reaction diffusion equations, Springer-Verlag, New York, 1983.

42. P. K. Sweby, High resolution schemes using flux limiters for hyperbolic conservation laws, SIAM J. Numer. Anal. 21 (1984), 995-1011.

43. A. Szepessy, Convergence of a shock-capturing streamline diffusion finite element method for a scalar conservation law in two space dimensions, Math. Comp. 53 (1989), 527--545.

44. _ Convergence of the streamline diffusion finite element method for conservation laws, Thesis, Univ. of Göteborg, Sweden, 1989.

45. E. Tadmor, Numerical viscosity and the entropy condition for conservative difference schemes, Math. Comp. 43 (1984), 369-381.

46. __ Semi-discrete approximations to nonlinear systems of conservation laws; consistency and $L^{\infty}$-stability imply convergence, ICASE report no. 88-41 (1988).

47. L. Tartar, Compensated compactness and applications to partial differential equations, Research Notes in Math., Non Linear Analysis and Mechanics (R. J. Knops, ed.), Heriot-Watt Symposium, vol. 4, Pitman, New York, 1979.

48. __ The compensated compactness method applied to systems of conservation laws, Systems of Nonlinear P.D.E. (J. M. Ball, ed.), NATO ASI Series, Reidel, 1983, pp. 263-285.

49. B. Van Leer, Towards the ultimate conservative difference scheme, IV: a new approach to numerical convection, J. Comput. Phys. 23 (1977), 276-299.

50. J. P. Vila, High-order schemes and entropy condition for nonlinear hyperbolic systems of conservation laws, Math. Comp. 50 (1988), 53-73.

51. A. I. Volpert, The space BV and quasilinear equations, Math. USSR-Sb. 2 (1967), 257-267.

52. Wang Jing-Hua, Li Xue-Wu, and Huang Jin-Yang, Lax-Friedrichs difference approximations to isentropic equations of gas dynamics, Preprint, Institute of Systems Science, Academia Sinica, Beijing, China.

Centre de Mathématiques Appliquées, Unité Associée au CNRS, URAD 0756, Ecole Polytechnique, 91128 Palaiseau Cedex, France

Current address, Ph. Le Floch: Courant Institute of Mathematical Sciences, New York University, 251 Mercer St., New York, New York 10012 